\title{
Convergence of a Higher-Order Vortex Method for Two-Dimensional Euler Equations*
}

\author{
By C. Chiu and R. A. Nicolaides
}

\begin{abstract}
There has been considerable interest recently in the convergence properties of point vortex methods. In this paper, we define a vortex method using vortex multipoles and obtain error estimates for it. In the case of a nonuniform mesh, the rate of convergence of the dipolar algorithm is shown to be of higher order of accuracy than obtained with the simple vortex methods.
\end{abstract}

1. Introduction. Although vortex methods have been used for many years for approximation of the partial differential equations of incompressible inviscid fluid dynamics, [12], [13], [15], a precise mathematical analysis was not available until very recently. In fact, the first complete analysis of a two-dimensional vortex method was given by Hald [10] in 1979. Since then, many papers have appeared giving error estimates for two-dimensional and three-dimensional vortex methods, including [1], [2], [3], [4], [5] and [18]. These analyses mostly assume a uniform mesh for the initial vorticity discretization. As a result of the mesh uniformity, the resulting error estimates are of unexpectedly high order of accuracy, being limited essentially by the regularity of the initial vorticity distribution. In more realisitic situations, it is improbable that uniform meshes can be used, e.g., if there are irregular bodies in the flow. In this case, the accuracy of the standard vortex methods will drop to first or second order, regardless of the initial regularity. In order to deal with nonuniform meshes, [17] defines some new vortex schemes for the two-dimensional incompressible Euler equations. In this paper we shall give a complete error estimate for one of them. This method yields higher order of accuracy even on nonuniform meshes. This is achieved by using not only the usual $\delta$ function point vortices, but also derivatives of such distributions.

In the next section we will define the algorithm and give explicit formulas for its implementation. Then, a rigorous error estimate will be provided following the Sobolev space technique of [5] and [18].

\section{The Construction of a Higher-Order Vortex Method.}

2-D Euler Equations. Let $u(x, t)=\left(u_{1}(x, t), u_{2}(x, t)\right), x \in \mathbf{R}^{2}$ and $t \in[0, \infty)$, be the velocity field and $w=\operatorname{curl} u=\partial_{1} u_{2}-\partial_{2} u_{1}$ be the vorticity. Assume that the exterior forces acting on the fluid are potential. Then the Euler equations are:

$$
\left\{\begin{array}{l}
w_{t}+(u \cdot \nabla) w=D w / D t=0, \quad w(x, 0)=w_{0}(x), x \in \mathbf{R}^{2}, \\
\operatorname{div} u=0 \\
u \rightarrow 0 \text { as }|x| \rightarrow \infty
\end{array}\right.
$$

Received August 8, 1986; revised October 13, 1987 and February 24, 1988.

1980 Mathematics Subject Classification (1985 Revision). Primary 65N30.

* This work was supported by the U. S. Air Force, under grant AFOSR 84-0137. 
Here, by definition, $D / D t:=\partial_{t}+(u \cdot \nabla)$ and is the usual material derivative. Concerning the existence and the uniqueness of solutions to the equation (2.1), see [14], [16], [19]. Smooth solutions are known to exist for all time in the twodimensional case with smooth initial data. In this paper we assume that the initial vorticity $w_{0}(x)$ of $(2.1)$ is smooth so that there exists a smooth solution of $(2.1)$ on some space-time interval $\mathbf{R}^{2} \times[0, T]$.

Let $X(x, t)$ be the path followed by a fluid particle which is at the position $x$ when $t=0$. Then the map $x \rightarrow X(x, t)$ satisfies the system of ordinary differential equations

$$
\frac{d X(x, t)}{d t}=u(X(x, t), t), \quad X(x, 0)=x .
$$

Then, (2.1) with the initial vorticity $w(x, 0)=w_{0}(x)$ satisfies $w(X(x, t), t)=$ $w_{0}(x)$. In this paper we only consider those flows with smooth vorticity $w_{0}(x)$ which have compact support. Under this assumption, there exists a bounded set $\Omega$ such that $\operatorname{supp} w(\cdot, t) \subset \Omega, \forall t \in[0, T]$.

Vortex Methods. Vortex methods are based upon the tracking of finite numbers of fluid particles and evaluating velocities by discretizing certain singular integrals. The basic idea of vortex methods is to approximate the initial vorticity by a linear combination of Dirac delta functions. For example, approximate $w_{0}$ by $w_{0}^{h}=$ $\sum_{j \in J} \alpha_{j} \delta\left(x-x_{j}\right)$ where $\alpha_{j} \in \mathbf{R}$.

By following those particles whose positions at $t=0$ are $\left\{x_{j}\right\}_{j \in J}$, using (2.2) with $x=x_{j}$, we get $w^{h}(x, t)=\sum_{j \in J} \alpha_{j} \delta\left(x-X\left(x_{j}, t\right)\right)$.

To compute $u$, one uses the fact that $\operatorname{div} u=0$ to introduce a stream function from which the velocity may be expressed as a singular integral. The singular kernel is then smoothed by a cutoff function, and quadrature rules are then needed to evaluate the integral. In order to get arbitrarily high order of accuracy by the above method, a uniform mesh has to be assumed. It can be obtained, for example, by subdividing the plane into squares of side $h$ and letting $\left\{x_{j}\right\}_{j \in J}$ be the corner points of the squares [18]. We will now introduce our algorithm and some related theorems. This algorithm allows us to deal with nonuniform meshes and still obtain high-order accuracy.

A Higher-Order Vortex Method. Recall that if the initial vorticity function is smooth, then the classical solution of $(2.1)$ is given by $w(X(x, t), t)=w_{0}(x)$. Now, let $\phi(\cdot) \in \mathscr{D}\left(\mathbf{R}^{2}\right)$ where $\mathscr{D}\left(\mathbf{R}^{2}\right)=\left\{\phi(\cdot) \in C^{\infty}\left(\mathbf{R}^{2}\right) \mid \phi(\cdot)\right.$ has compact support $\}$; $\mathscr{D}^{\prime}\left(\mathbf{R}^{2}\right)$ is the dual space of $\mathscr{D}\left(\mathbf{R}^{2}\right)$ and $\langle\cdot, \cdot\rangle$ denotes the duality pairing. Then,

$$
\langle w(\cdot, t), \phi(\cdot)\rangle=\int_{\mathbf{R}^{2}} w(x, t) \phi(x) d x=\int_{\mathbf{R}^{2}} w_{0}(x) \phi(X(x, t)) d x=\left\langle w_{0}(\cdot), \phi(X(\cdot, t))\right\rangle,
$$

because the determinant of the Jacobian matrix of $x \rightarrow X$ is 1 since div $u=0$. Thus, we define a weak solution of the Euler equation as follows:

Definition 2.1. Assume that a unique solution of (2.2) exists. For $w(\cdot, t) \in$ $\mathscr{D}^{\prime}\left(\mathbf{R}^{2}\right)$ and $w_{0}(\cdot) \in \mathscr{D}^{\prime}\left(\mathbf{R}^{2}\right)$, if

$$
\langle w(\cdot, t), \phi(\cdot)\rangle=\left\langle w_{0}(\cdot), \phi(X(\cdot, t))\right\rangle \quad \forall \phi(\cdot) \in \mathscr{D}\left(\mathbf{R}^{2}\right),
$$

then, $w(\cdot, t)$ is said to be the weak vorticity of the Euler equation (2.1). 
THEOREM 2.1. Suppose that $X\left(x_{0}, t\right)$ exists.

If $w_{0}(x)=a \delta\left(x-x_{0}\right)+b \delta_{x_{1}}\left(x-x_{0}\right)+c \delta_{x_{2}}\left(x-x_{0}\right)$, where $a, b$ and $c$ are constants, then the weak vorticity as defined above is

$$
w(x, t)=a \delta\left(x-X\left(x_{0}, t\right)\right)+b(t) \delta_{x_{1}}\left(x-X\left(x_{0}, t\right)\right)+c(t) \delta_{x_{2}}\left(x-X\left(x_{0}, t\right)\right),
$$

where $\delta_{x_{1}}$ and $\delta_{x_{2}}$ are derivatives of the Dirac Delta function $\delta$ and

$$
\left(\begin{array}{l}
b(t) \\
c(t)
\end{array}\right)=M\left(x_{0}, t\right)\left(\begin{array}{l}
b \\
c
\end{array}\right), \quad M\left(x_{0}, t\right)=\left(\frac{d X_{i}}{d x_{j}}\right)
$$

is the Jacobian matrix of $x \rightarrow X$ at $x_{0}$.

Remark. Note that

$$
\frac{d}{d t} M\left(x_{0}, t\right)=\frac{d}{d t}\left(\frac{d X_{i}}{d x_{j}}\right)=\left(\frac{d u_{i}}{d x_{j}}\right)=\left(\frac{d u_{i}}{d X_{k}} \cdot \frac{d X_{k}}{d x_{j}}\right),
$$

using the summation convention. So, $M\left(x_{0}, t\right)$ satisfies the following system of ordinary differential equations:

$$
\left\{\begin{array}{l}
\frac{d M}{d t}=\nabla u \cdot M \\
M\left(x_{0}, 0\right)=I
\end{array}\right.
$$

where $M=M\left(x_{0}, t\right)$ and $u=u\left(X\left(x_{0}, t\right), t\right)$.

Proof. For all $\phi \in \mathscr{D}\left(\mathbf{R}^{2}\right)$ we have

$$
\begin{aligned}
\left\langle w_{0}(\cdot)\right. & , \phi(X(\cdot, t))\rangle=a \phi\left(X\left(x_{0}, t\right)\right)-b d \phi\left(X\left(x_{0}, t\right)\right) / d x_{1}-c d \phi\left(X\left(x_{0}, t\right)\right) / d x_{2} \\
= & a \phi\left(X\left(x_{0}, t\right)\right)-b\left[\left(d \phi / d X_{1}\right)\left(d X_{1} / d x_{1}\right)+\left(d \phi / d X_{2}\right)\left(d X_{2} / d x_{1}\right)\right] \\
& -c\left[\left(d \phi / d X_{1}\right)\left(d X_{1} / d x_{2}\right)+\left(d \phi / d X_{2}\right)\left(d X_{2} / d x_{2}\right)\right] \quad\left(\text { at } x_{0}\right) \\
= & a \phi\left(X\left(x_{0}, t\right)\right)-b(t) d \phi\left(X\left(x_{0}, t\right)\right) / d X_{1}-c(t) d \phi\left(X\left(x_{0}, t\right)\right) / d X_{2} \\
= & \left\langle a \delta\left(\cdot-X\left(x_{0}, t\right)\right)+b(t) \delta_{x_{1}}\left(\cdot-X\left(x_{0}, t\right)\right)+c(t) \delta_{x_{2}}\left(\cdot-X\left(x_{0}, t\right)\right), \phi(\cdot)\right\rangle \\
= & \langle w(\cdot, t), \phi(\cdot)\rangle .
\end{aligned}
$$

Using Definition $2.1, w(\cdot, t)$ is the weak vorticity.

Now we will define our vortex method by specifying $a, b$ and $c$ over an initial vortex distribution. This can be done in many ways [17]. The method used below is based on direct numerical integration.

The Vorticity Field.
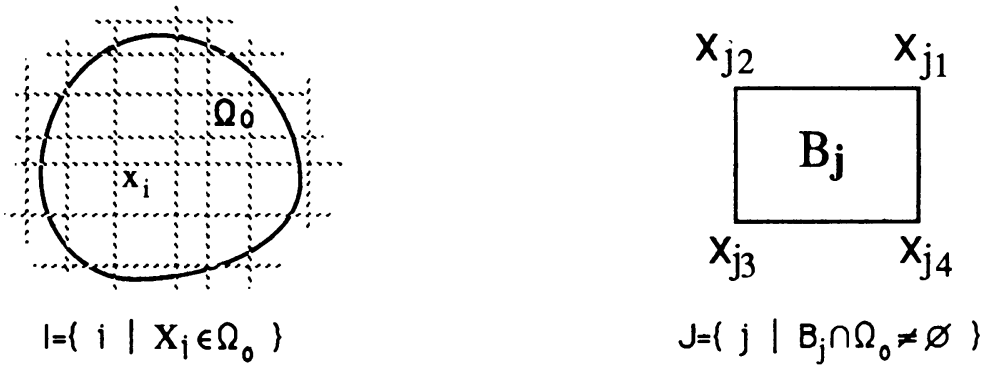

FIGURE 1 
Take an arbitrarily spaced rectangular mesh on $\mathbf{R}^{2}$. Let supp $w_{0} \subset \Omega_{0}$ which is bounded. To each rectangle formed by adjacent coordinate lines, assign an index $j$ and denote it by $B_{j}$. Denote the lengths of the edges of $B_{j}$ by $h_{j 1}$ and $h_{j 2}$. Let $\left\{x_{j k}\right\}_{k=1,4}$ be the four corners of $B_{j}$. To each corner of $B_{j} \cap \Omega_{0} \neq \varnothing$, assign a global index $i$, as shown in Figure 1.

Interpreting the initial vorticity $w_{0}$ as a distribution, we shall approximate $w_{0}$ by another distribution $w_{0}^{h}$ of the form

$$
\begin{aligned}
w_{0}^{h}(x) & =\sum_{j \in J} \sum_{k=1,4}\left[a_{j k} \delta\left(x-x_{j k}\right)+b_{1 j k} \delta_{x_{1}}\left(x-x_{j k}\right)+b_{2 j k} \delta_{x_{2}}\left(x-x_{j k}\right)\right] \\
& =\sum_{i \in I}\left[c_{i} \delta\left(x-x_{i}\right)+d_{1 i} \delta_{x_{1}}\left(x-x_{i}\right)+d_{2 i} \delta_{x_{2}}\left(x-x_{i}\right)\right]
\end{aligned}
$$

Then, based on Theorem 2.1, we expect that $w(\cdot, t)$ can be approximated by

$$
\begin{aligned}
w^{h}(x) & =\sum_{j \in J} \sum_{k=1,4}\left[a_{j k} \delta\left(x-X\left(x_{j k}, t\right)\right)+b_{1 j k}(t) \delta_{x_{1}}\left(x-X\left(x_{j k}, t\right)\right)\right. \\
& \left.\quad+b_{2 j k}(t) \delta_{x_{2}}\left(x-X\left(x_{j k}, t\right)\right)\right] \\
& =\sum_{i \in I}\left[c_{i} \delta\left(x-X\left(x_{i}, t\right)\right)+d_{1 i}(t) \delta_{x_{1}}\left(x-X\left(x_{i}, t\right)\right)+d_{2 i}(t) \delta_{x_{2}}\left(x-X\left(x_{i}, t\right)\right)\right]
\end{aligned}
$$

where

$$
\left(\begin{array}{l}
b_{1 j k}(t) \\
b_{2 j k}(t)
\end{array}\right)=M\left(x_{j k}, t\right)\left(\begin{array}{l}
b_{1 j k} \\
b_{2 j k}
\end{array}\right) \text { and }\left(\begin{array}{l}
d_{1 i}(t) \\
d_{2 i}(t)
\end{array}\right)=M\left(x_{i}, t\right)\left(\begin{array}{l}
d_{1 i} \\
d_{2 i}
\end{array}\right) .
$$

Concerning the choice of the coefficients $\left\{a_{j k}, b_{1 j k}, b_{2 j k}\right\}$, observe that for $\phi(\cdot) \in$ $\mathscr{D}\left(\mathbf{R}^{2}\right)$,

$$
\left\langle w_{0}^{h}(\cdot), \phi(\cdot)\right\rangle=\sum_{j \in J} \sum_{k=1,4}\left[a_{j k} \phi\left(x_{j k}\right)-b_{1 j k}(t) \phi_{x_{1}}\left(x_{j k}\right)-b_{2 j k}(t) \phi_{x_{2}}\left(x_{j k}\right)\right],
$$

whereas

$$
\left\langle w_{0}(\cdot), \phi(\cdot)\right\rangle=\int_{\mathbf{R}^{2}} w_{0}(x) \phi(x) d x=\sum_{j \in J} \int_{B j} w_{0}(x) \phi(x) d x .
$$

This suggests that approximating $w_{0}$ by $w_{0}^{h}$ corresponds to approximating the integral

$$
\int_{B j} w_{0}(x) \phi(x) d x
$$

by some numerical integration rule, where $\left\{a_{j k}, b_{1 j k}, b_{2 j k}\right\}_{j \in J, k=1,4}$ define this rule. For our algorithm, the following quadrature rule is used [17]. For a 2-dimensional rectangle $B$ with corners $\left\{P_{i}\right\}_{i=1,4}$, sides $h_{1}$ and $h_{2}$, as shown in Figure 2, and $f \in C^{1}(B)$,

$$
\begin{aligned}
\int_{B} f(x) d x \approx & \frac{h_{1} h_{2}}{4}\left(f\left(P_{1}\right)+f\left(P_{2}\right)+f\left(P_{3}\right)+f\left(P_{4}\right)\right) \\
& +\frac{h_{1}^{2} h_{2}}{24}\left[-f_{x}\left(P_{1}\right)+f_{x}\left(P_{2}\right)+f_{x}\left(P_{3}\right)-f_{x}\left(P_{4}\right)\right] \\
& +\frac{h_{1} h_{2}^{2}}{24}\left[-f_{y}\left(P_{1}\right)-f_{y}\left(P_{2}\right)+f_{y}\left(P_{3}\right)+f_{y}\left(P_{4}\right)\right]
\end{aligned}
$$




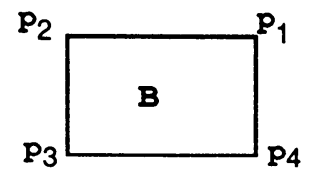

FIGURE 2

For this rule, a direct calculation shows:

THEOREM 2.2. The quadrature rule $(*)$ is exact for all third-degree polynomials.

Denote the right-hand side of $(*)$ by $Q_{2}(B, f)$, i.e., $\int_{B} f(x) d x \approx Q_{2}(B, f)$. Thus, if $\left\{a_{j k}, b_{1 j k}, b_{2 j k}\right\}_{j \in J, k=1,4}$ are chosen by the above rule, then for $\phi(\cdot) \in \mathscr{D}\left(\mathbf{R}^{2}\right)$,

$$
\begin{aligned}
\left\langle w_{0}^{h}(\cdot), \phi(\cdot)\right\rangle & =\sum_{j \in J} \sum_{k=1,4}\left[a_{j k} \phi\left(x_{j k}\right)-b_{1 j k} \phi_{x_{1}}\left(x_{j k}\right)-b_{2 j k} \phi_{x_{2}}\left(x_{j k}\right)\right] \\
& =\sum_{j \in J} Q_{2}\left(B_{j}, \phi\right) .
\end{aligned}
$$

Then, when $h \rightarrow 0, \sum_{j \in J} Q_{2}\left(B_{j}, \phi\right) \rightarrow\left\langle w_{0}(\cdot), \phi(\cdot)\right\rangle$. More precisely, $w_{0}^{h}$ converges in $\mathscr{D}^{\prime}\left(\mathbf{R}^{2}\right)$ to $w_{0}$ as $h \rightarrow 0$. Correspondingly, $w^{h} \rightarrow w$ in $\mathscr{D}^{\prime}\left(\mathbf{R}^{2}\right)$, by Definition 2.1. To see what the corresponding coefficients $\left\{c_{i}, d_{1 i}, d_{2 i}\right\}$ are, let us consider an example.

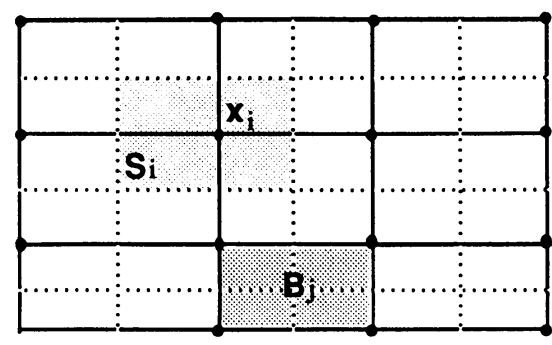

FIGURE 3

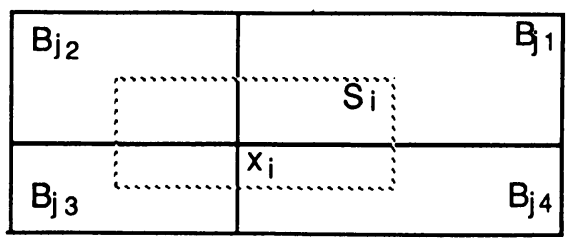

FIGURE 4

Suppose that the mesh is as shown in Figure 3. The points with closed circles are the nodal points of the mesh. Then $B_{j}$ is some rectangle with nodal points as its four corners while $S_{i}$ is a rectangle which contains the nodal point $x_{i}$ and is bounded by dotted lines. Note that dotted lines equally divide sides of every $B_{j} . B_{j 1}, B_{j 2}, B_{j 3}$ and $B_{j 4}$, as shown in Figure 4 , are four adjacent rectangles. Let $S_{i}$ be the rectangle shown and $\left|S_{i}\right|$ be its area. Since $x_{i}$ is a common corner of $B_{j 1}, B_{j 2}, B_{j 3}$ and $B_{j 4}$, by the quadrature rule $(*)$, we have the following:

$$
\begin{aligned}
c_{i} \phi\left(x_{i}\right) & -d_{1 i} \phi_{x_{1}}\left(x_{i}\right)-d_{2 i} \phi_{x_{2}}\left(x_{i}\right) \\
= & (1 / 4)\left[h_{j_{11}} h_{j_{12}}+h_{j_{21}} h_{j_{22}}+h_{j_{31}} h_{j_{32}}+h_{j_{41}} h_{j_{42}}\right] w_{0}\left(x_{i}\right) \phi\left(x_{i}\right) \\
& +(1 / 24)\left[h_{j_{11}}^{2} h_{j_{12}}-h_{j_{21}}^{2} h_{j_{22}}-h_{j_{31}}^{2} h_{j_{32}}+h_{j_{41}}^{2} h_{j_{42}}\right]\left(w_{0} \phi\right)_{x_{1}}\left(x_{i}\right) \\
& +(1 / 24)\left[h_{j_{11}} h_{j_{12}}^{2}+h_{j_{21}} h_{j_{22}}^{2}-h_{j_{31}} h_{j_{32}}^{2}-h_{j_{41}} h_{j_{42}}^{2}\right]\left(w_{0} \phi\right)_{x_{2}}\left(x_{i}\right) \\
= & \left|S_{i}\right|\left\{w_{0}\left(x_{i}\right) \phi\left(x_{i}\right)+H_{i 1}\left(w_{0} \phi\right)_{x_{1}}\left(x_{i}\right)+H_{i 2}\left(w_{0} \phi\right)_{x_{2}}\left(x_{i}\right)\right\} .
\end{aligned}
$$


Assume that there exists a constant $C>0$ such that

$$
\frac{\max _{j \in J}\left(h_{j 1}, h_{j 2}\right)}{\min _{j \in J}\left(h_{j 1}, h_{j 2}\right)} \leq C
$$

and let $h=\max _{j \in J}\left(h_{i 1}, h_{j 2}\right)$; then, $H_{i 1}=O(h)$ and $H_{i 2}=O(h)$ and

$$
\begin{gathered}
c_{i}=\left|S_{i}\right|\left\{w_{0}\left(x_{i}\right)+H_{i 1} w_{0 x_{1}}\left(x_{i}\right)+H_{i 2} w_{0 x_{2}}\left(x_{i}\right)\right\}, \\
d_{1 i}=-\left|S_{i}\right| H_{i 1} w_{0}\left(x_{i}\right), \quad d_{2 i}=-\left|S_{i}\right| H_{i 2} w_{0}\left(x_{i}\right), \quad \forall i \in I .
\end{gathered}
$$

The Velocity Field. In order to obtain the velocity field from the vorticity field, we need the following result. Let $K: \mathbf{R}^{2} \rightarrow \mathbf{R}^{2}$ be defined by

$$
K=\frac{1}{2 \pi|x|^{2}}\left(\begin{array}{c}
-x_{2} \\
x_{1}
\end{array}\right) \text {. }
$$

LEMMA 2.1. The convolution operator $f \rightarrow K * f$ is a bounded linear mapping from $L^{\infty}\left(\mathbf{R}^{2}\right) \cap L^{1}\left(\mathbf{R}^{2}\right)$ into $B^{0}\left(\mathbf{R}^{2}\right)^{2}$ (set of bounded and continuous $2-\mathrm{D}$ vectorvalued functions). Moreover, if $f \in L^{\infty}\left(\mathbf{R}^{2}\right) \cap L^{1}\left(\mathbf{R}^{2}\right)$ and satisfies $f(x) \rightarrow 0$ as $|x| \rightarrow \infty$, then the function $v=K * f$ (2-D vector) is the unique solution of

Proof. See [16].

$$
\left\{\begin{array}{l}
\operatorname{div} v=0 \\
\operatorname{curl} v=f \\
v(x) \rightarrow 0 \text { as }|x| \rightarrow \infty, x \in \mathbf{R}^{2}
\end{array}\right.
$$

It follows from Lemma 2.1 that $u(\cdot, t)=K * w(\cdot, t)$ in problem (2.1). After computing the approximate vorticity field $w^{h}$, we need to find the corresponding velocity field. It would seem natural to set $u^{h}(\cdot, t)=K * w^{h}(\cdot, t)$, but since the kernel $K$ is a singular function, its convolution with delta functions is not defined in general. To avoid this problem, the now standard remedy is to regularize $K$ as follows.

Let $f(x): \mathbf{R}^{2} \rightarrow \mathbf{R}$ satisfy $\int_{\mathbf{R}^{2}} f(x) d x=1$ and let $f_{\varepsilon}(x)=\left(1 / \varepsilon^{2}\right) f(x / \varepsilon) . f$ and $f_{\varepsilon}$ are referred to as "cutoff" functions. If $K_{\varepsilon}=K * f_{\varepsilon}$, then Lemma 2.1 implies that $K_{\varepsilon} \in B^{1}\left(\mathbf{R}^{2}\right)^{2}$ provided $f \in W^{1, \infty}\left(\mathbf{R}^{2}\right) \cap W^{1,1}\left(\mathbf{R}^{2}\right)$. Then, $u(\cdot, t)$ will be approximated by $u_{\varepsilon}^{h}=w^{h} * K_{\varepsilon}$, so that

$$
\begin{array}{r}
u_{\varepsilon}^{h}(x)=\sum_{j \in J} \sum_{k=1,4}\left[a_{j k} K_{\varepsilon}\left(x-X\left(x_{j k}, t\right)\right)-b_{1 j k}(t) K_{\varepsilon x_{1}}\left(x-X\left(x_{j k}, t\right)\right)\right. \\
\left.-b_{2 j k}(t) K_{\varepsilon x_{2}}\left(x-X\left(x_{j k}, t\right)\right)\right] \\
=\sum_{i \in I}\left[c_{i} K_{\varepsilon}\left(x-X\left(x_{i}, t\right)\right)-d_{1 i}(t) K_{\varepsilon x_{1}}\left(x-X\left(x_{i}, t\right)\right)\right. \\
\left.-d_{2 i}(t) K_{\varepsilon x_{2}}\left(x-X\left(x_{i}, t\right)\right)\right] .
\end{array}
$$

Here, $K_{\varepsilon x_{1}}=d K_{\varepsilon} / d x_{1}, K_{\varepsilon x_{2}}=d K_{\varepsilon} / d x_{2}$ and $\left\{X\left(x_{i}, t\right)\right\}_{i \in I}$ are exact particle positions at $t$. But we can compute only approximate positions $\left\{X^{h}\left(x_{i}, t\right)\right\}_{i \in I}$ and corresponding approximate Jacobian matrices $\left\{M^{h}\left(x_{i}, t\right)\right\}_{i \in I}$. Therefore, only approximate coefficients $\left\{d_{1 i}(t), d_{2 i}(t)\right\}_{i \in I}$ can be obtained. So, the actual velocity field we compute is

$$
\begin{aligned}
\mathscr{U}_{\varepsilon}^{h}(x, t)=\sum_{i \in I}\left[c_{i} K_{\varepsilon}\left(x-X^{h}\left(x_{i}, t\right)\right)-d_{1 i}(t)\right. & K_{\varepsilon x_{1}}\left(x-X^{h}\left(x_{i}, t\right)\right) \\
& \left.-d_{2 i}(t) K_{\varepsilon x_{2}}\left(x-X^{h}\left(x_{i}, t\right)\right)\right] .
\end{aligned}
$$

Here we use $\mathscr{U}$ to denote the numerical velocity field. 
In summary, the 2-D algorithm is as follows:

$$
\left\{\begin{array}{l}
\frac{d X^{h}(x, t)}{d t}=\mathscr{U}_{\varepsilon}^{h}\left(X^{h}, t\right), \quad X^{h}(x, 0)=x, \\
\frac{d M^{h}(x, t)}{d t}=\nabla \mathscr{U}_{\varepsilon}^{h}\left(X^{h}, t\right) M^{h}(x, t), \quad M^{h}(x, 0)=I
\end{array}\right.
$$

(see the remark after Theorem 2.1 ), where $\mathscr{U}_{\varepsilon}^{h}$ is the numerical velocity field given by $(2.5), X^{h}(x, t)$ is the computed particle position at $t$ with its initial position at $x$, and $M^{h}(x, t)=\left(\partial X_{i}^{h} / \partial x_{j}\right)$ is the Jacobian matrix of the mapping $x \rightarrow X^{h}(x, t)$. Moreover,

$$
\left(\begin{array}{l}
d_{1 i}(t) \\
d_{2 i}(t)
\end{array}\right)=M^{h}\left(x_{i}, t\right)\left(\begin{array}{l}
d_{1 i} \\
d_{2 i}
\end{array}\right)
$$

and $\left\{c_{i}, d_{1 i}, d_{2 i}\right\}_{i \in I}$ are given by $(2.4)$.

3. Error Estimates. In this section we will give a complete error estimate for the algorithm constructed in the last section. The analysis given here consists of two parts, one for estimating the consistency error and the other for stability error. The first part is based upon Sobolev space theory. The second part depends on analysis of the velocity kernel and the behavior of the cutoff function.

Notations and Definitions. The norms used for the analysis are discrete $L^{p_{-}}$ norms.

Definition 3.1. For $f(\cdot) \in\left[L^{p}(\Omega)\right]^{2}$ or $\left[L^{p}(\Omega)\right]^{2 \times 2}$, define

$$
\|f\|_{h, p}=\left[\sum_{i \in I}\left|f\left(x_{i}\right)\right|^{p} h^{2}\right]^{1 / p} .
$$

Let $x \rightarrow X(x, t)$ be the trajectory mapping and $x \rightarrow X^{h}(x, t)$ be the computed trajectory mapping. For $F\left(X(\cdot, t), X^{h}(\cdot, t), t\right) \in\left[L^{p}(\Omega)\right]^{2}$ or $\left[L^{p}(\Omega)\right]^{2 \times 2}$, define

$$
\|F(t)\|_{h, p}=\left[\sum_{i \in I}\left|F\left(X\left(x_{i}, t\right), X^{h}\left(x_{i}, t\right), t\right)\right|^{p} h^{2}\right]^{1 / p} .
$$

Let $e\left(X(\cdot, t), X^{h}(\cdot, t), t\right)=X(\cdot, t)-X^{h}(\cdot, t)$, and $E\left(X(\cdot, t), X^{h}(\cdot, t), t\right)=M(\cdot, t)-$ $M^{h}(\cdot, t)$.

For the error estimate, we define $w^{h}(x)$ and $w^{h}(x)$ as

$$
\begin{aligned}
& w^{h}(x)=\sum_{j \in J} \sum_{k=1,4}\left[a_{j k} \delta\left(x-X\left(x_{j k}, t\right)\right)+b_{1 j k}(t) \delta_{x_{1}}\left(x-X\left(x_{j k}, t\right)\right)\right. \\
& \left.+b_{2 j k}(t) \delta_{x_{2}}\left(x-X\left(x_{j k}, t\right)\right)\right] \\
& =\sum_{i \in I}\left[c_{i} \delta\left(x-X\left(x_{i}, t\right)\right)+d_{1 i}(t) \delta_{x_{1}}\left(x-X\left(x_{i}, t\right)\right)+d_{2 i}(t) \delta_{x_{2}}\left(x-X\left(x_{i}, t\right)\right)\right], \\
& \omega^{h}(x)=\sum_{j \in J} \sum_{k=1,4}\left[a_{j k} \delta\left(x-X^{h}\left(x_{j k}, t\right)\right)+b_{1 j k}(t) \delta_{x_{1}}\left(x-X^{h}\left(x_{j k}, t\right)\right)\right. \\
& \left.+b_{2 j k}(t) \delta_{x_{2}}\left(x-X^{h}\left(x_{j k}, t\right)\right)\right] \\
& =\sum_{i \in I}\left[c_{i} \delta\left(x-X^{h}\left(x_{i}, t\right)\right)+d_{1 i}(t) \delta_{x_{1}}\left(x-X^{h}\left(x_{i}, t\right)\right)\right. \\
& \left.+d_{2 i}(t) \delta_{x_{2}}\left(x-X^{h}\left(x_{i}, t\right)\right)\right] \text {. }
\end{aligned}
$$


Then Table 1 contains all quantities we will use for the error estimate.

TABLE 1

\begin{tabular}{lccc}
\hline & Trajectory & Vorticity & Velocity \\
\hline $\begin{array}{l}\text { Exact } \\
\text { Solution }\end{array}$ & $x \rightarrow X(x, t)$ & $w(x, t)$ & $u=(K * w)$ \\
\hline $\begin{array}{c}\text { Computed } \\
\text { Solution }\end{array}$ & $x \rightarrow X^{h}(x, t)$ & $\omega^{h}(x, t)$ & $\mathscr{U}_{\varepsilon}^{h}=K_{\varepsilon} * w^{h}$ \\
\hline $\begin{array}{l}\text { Intermediate } \\
\text { Quantity }\end{array}$ & & $w^{h}(x, t)$ & $u_{\varepsilon}=K_{\varepsilon} * w$ \\
\hline
\end{tabular}

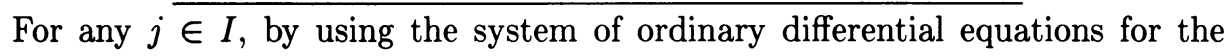
particle trajectories, we have

$$
\begin{aligned}
& \frac{d X\left(x_{j}, t\right)}{d t}-\frac{d X^{h}\left(x_{j}, t\right)}{d t}=u\left(X\left(x_{j}, t\right), t\right)-\mathscr{U}_{\varepsilon}^{h}\left(X^{h}\left(x_{j}, t\right), t\right) \\
& \quad=\left[u\left(X\left(x_{j}, t\right), t\right)-u_{\varepsilon}^{h}\left(X\left(x_{j}, t\right), t\right)\right]+\left[u_{\varepsilon}^{h}\left(X\left(x_{j}, t\right), t\right)-\mathscr{U}_{\varepsilon}^{h}\left(X^{h}\left(x_{j}, t\right), t\right)\right],
\end{aligned}
$$

where the first bracketed expression is called the consistency error, and the second the stability error.

The Consistency Error. Let

$$
\mathrm{I}(\cdot, t)=u(\cdot, t)-u_{\varepsilon}(\cdot, t)=K * w-K_{\varepsilon} * w
$$

and

$$
\mathrm{II}(\cdot, t)=u_{\varepsilon}(\cdot, t)-u_{\varepsilon}^{h}(\cdot, t)=K_{\varepsilon} * w-K_{\varepsilon} * w^{h} .
$$

Then, the consistency error is I + II. For I, we have the following result.

THEOREM 3.1. Assume that there exists an integer $k \geq 1$ such that

(i) $\int_{\mathbf{R}^{2}} f(x) d x=1$,

(ii) $\int_{\mathbf{R}^{2}} x^{\alpha} f(x) d x=0, \forall \alpha \in N^{2}, 1 \leq|\alpha| \leq k-1$,

(iii) $\int_{\mathbf{R}^{2}}|x|^{k}|f(x)| d x<\infty$.

Then there exists a constant $C=C\left(p, T, w_{0}\right)>0$ such that $\|\mathrm{I}(\cdot, t)\|_{L^{\infty}\left(\mathbf{R}^{2}\right)} \leq C \varepsilon^{k}$ and $\|\mathrm{I}(t)\|_{h, p} \leq C \varepsilon^{k}$ for all $p \in[1, \infty], t \in[0, T]$.

Proof. See [18, Chapter II, Lemma 4.1].

In order to analyze $\mathrm{II}(\cdot, t)$, we need to discuss a few auxiliary results. First of all, consider some properties of the regularized kernel $K_{\varepsilon}$. These properties are also very useful for the stability error estimate. We begin by recalling a classical result.

LEMMA 3.1 (Calderon-Zygmund). The convolution operator $f \rightarrow\left(\partial K / \partial x_{i}\right) * f$ is a bounded linear mapping from $L^{p}\left(\mathbf{R}^{2}\right)$ into $\left[L^{p}\left(\mathbf{R}^{2}\right)\right]^{2}$, for $i=1,2$ and $1<p<$ $\infty$.

LEMMA 3.2. Let $p \in(1, \infty)$ and $f \in W^{\ell-1, p}\left(\mathbf{R}^{2}\right)$ for some integer $\ell \geq 1$. Then there exists some constant $C$ such that

$$
\left\|\partial^{\alpha} K_{\varepsilon}\right\|_{L^{p}\left(\mathbf{R}^{2}\right)} \leq \frac{C}{\varepsilon^{\ell-1+(2 / q)}},
$$

for $\alpha \in N^{2}$ with $|\alpha|=\ell$ and $(1 / p)+(1 / q)=1$.

Proof. See [18, Chapter II, Lemma 3.2(ii)]. 
LEMMA 3.3. Let $\ell$ be a nonnegative integer. The following properties hold for all $\alpha \in N^{2}$ with $|\alpha|=\ell$ :

(a) If $f \in W^{\ell, 1}\left(\mathbf{R}^{2}\right) \cap W^{\ell, \infty}\left(\mathbf{R}^{2}\right)$, we have $\left|\partial^{\alpha} K_{\varepsilon}(x)\right| \leq C_{1} / \varepsilon^{\ell+1}$ for all $x \in \mathbf{R}^{2}$.

(b) If $f \in W^{\ell, 1}\left(\mathbf{R}^{2}\right) \cap W^{\ell, \infty}\left(\mathbf{R}^{2}\right)$ satisfies in addition $|x|^{\ell+2}\left|\partial^{\alpha} f(x)\right| \leq C_{2}$, then

$$
\left|\partial^{\alpha} K_{\varepsilon}(x)\right| \leq \frac{C_{3}}{|x|^{\ell+1}} \quad \text { for all }|x| \geq \varepsilon .
$$

Proof. See [18, Chapter II, Lemma 3.3].

LEMMA 3.4. Let $S$ be a compact set in $\mathbf{R}^{2}$. For any multi-index $\beta$, assume that there exists a constant $C_{1}>0$ such that $|x|^{|\beta|+2}\left|\partial^{\beta} f(x)\right| \leq C_{1}$. Then there is a constant $C=C(S)$ such that for all $\varepsilon \leq \frac{1}{2}$,

$$
\left\|\partial^{\beta} K_{\varepsilon}\right\|_{L^{1}(S)} \leq \begin{cases}C(S), & |\beta|=0 \\ C(S)|\log \varepsilon|, & |\beta|=1 \\ C(S) \varepsilon^{1-|\beta|}, & |\beta|>1\end{cases}
$$

Proof. Let $B_{\varepsilon}=\left\{x \in \mathbf{R}^{n}|| x \mid<\varepsilon\right\}$. Then,

$$
\begin{aligned}
&\left\|\partial^{\beta} K_{\varepsilon}\right\|_{L^{1}(S)}=\int_{S}\left|\partial^{\beta} K_{\varepsilon}(x)\right| d x=\int_{S \cap B_{\varepsilon}}\left|\partial^{\beta} K_{\varepsilon}(x)\right| d x+\int_{S \backslash B_{\varepsilon}}\left|\partial^{\beta} K_{\varepsilon}(x)\right| d x \\
& \quad \leq C_{2} \varepsilon^{2} / \varepsilon^{|\beta|+1}+\int_{S \backslash B_{\varepsilon}} \frac{C_{3}}{|x|^{|\beta|+1}} d x \quad \text { (Lemma 3.3) } \\
& \quad \leq C_{2} \varepsilon^{1-|\beta|}+\int_{\varepsilon}^{\operatorname{diam}(S)} \frac{C_{4}}{|r|^{|\beta|} d r}|\beta|=0, \\
& \quad=C_{2} \varepsilon^{1-|\beta|}+C_{4} \begin{cases}{[\operatorname{diam}(S)-\varepsilon],} & |\beta|=1, \\
{[\log (\operatorname{diam}(S))-\log \varepsilon],} & |\beta|>1 . \square \\
(1-|\beta|)\left[\operatorname{diam}(S)^{1-|\beta|}-\varepsilon^{1-|\beta|}\right], & |\delta|\end{cases}
\end{aligned}
$$

As we defined in the last section, the initial coefficients of $\delta$ functions in the approximated vorticity field are chosen according to a quadrature rule. Now we want to find a bound for the error which results from the numerical integration. We first state a classical result due to Bramble and Hilbert [9, Theorem 4.1.3]. Let $k$ be a nonnegative integer, denote by $P_{k}$ the space of all polynomials of degree $\leq k$ in the $n$ variables $x_{1}, \ldots, x_{n}$.

LEMMA 3.5 (Bramble-Hilbert). Let $\Omega$ be an open bounded subset of $\mathbf{R}^{n}$ with a Lipschitz continuous boundary and let $L: \phi \rightarrow L(\phi)$ be a bounded linear functional on $W^{k, p}(\Omega), k \geq 1, p \in[1, \infty]$, with norm $\|L\|$, which satisfies $L(\phi)=0$ for all $\phi \in P_{k-1}$. Then there exists a constant $C>0$ such that

$$
|L(\phi)| \leq C\|L\||\phi|_{k, p, \Omega} \quad \forall \phi \in W^{k, p}(\Omega),
$$

where

$$
\|\phi\|_{k, p, \Omega}=\left(\sum_{|\alpha| \leq k} \int_{\Omega}\left|\partial^{\alpha} \phi\right|^{p} d x\right)^{1 / p}, \quad|\phi|_{k, p, \Omega}=\left(\sum_{|\alpha|=k} \int_{\Omega}\left|\partial^{\alpha} \phi\right| d x\right)^{1 / p}
$$

and $\|L\|=\sup _{\|\phi\|_{k, p, \Omega}=1}|L(\phi)|$. 
As a consequence of Lemma 3.5, we obtain

LEMMA 3.6. Let $j \in J$ and $B_{j}$ be a 2-D box as defined above. Assume that the center of $B_{j}$ is $x=\left(x_{1}, x_{2}\right) \in \mathbf{R}^{2}$. If $g(\cdot) \in W^{4,1}\left(B_{j}\right)$, then there exists a constant $C>0$ independent of $B_{j}$ such that

$$
\left|\int_{B_{j}} g(x) d x-Q_{2}\left(B_{j}, g\right)\right| \leq C h^{4}|g|_{4,1, B_{j}} .
$$

Proof. Let $\mathscr{B}=[-1,+1]^{2}$ and $B^{1}(\mathscr{B})$ be the set of functions whose derivatives through order one are bounded and continuous on $\mathscr{B}$. Then, for $g(\cdot) \in B^{1}(\mathscr{B})$, define $\mathscr{E}(g)=\int_{\mathscr{B}} g(x) d x-Q_{2}(\mathscr{B}, g)$. By Theorem $2.2, \mathscr{E}(g)=0$ for all $g \in$ $P_{3}(\mathscr{B})$.

It is very easy to check that $g \rightarrow \mathscr{E}(g)$ is a bounded linear functional on $B^{1}(\mathscr{B})$. By Sobolev's embedding theorem, $W^{4,1}(\mathscr{B}) \subset B^{1}(\mathscr{B})$. So, $g \rightarrow \mathscr{E}(g)$ is also a bounded linear functional on $W^{4,1}(\mathscr{B})$ which vanishes on $P_{3}(\mathscr{B})$. Thus, by the Bramble-Hilbert lemma, there is a constant $C_{1}>0$ such that

$$
|\mathscr{E}(g)| \leq C_{1}|g|_{4,1, \mathscr{B}}
$$

For a function $g(\cdot)$ defined on $B_{j}$, change variables by letting

$$
x_{i}=x_{i}+\left(h_{j i} / 2\right) \xi_{i}, \quad-1 \leq \xi_{i} \leq 1, i=1,2,
$$

and define $g(\xi)=g(x)=g\left(x_{1}+\left(h_{j 1} / 2\right) \xi_{1}, x_{2}+\left(h_{j 2} / 2\right) \xi_{2}\right)$. Then (3.1) follows from (3.2).

Now consider the second part of the consistency error. Recall that

$$
\begin{gathered}
\mathrm{II}(\cdot, t)=u_{\varepsilon}(\cdot, t)-u_{\varepsilon}^{h}(\cdot, t)=K_{\varepsilon} * w-K_{\varepsilon} * w^{h} \\
\mathrm{II}(X, t)=\left(K_{\varepsilon} * w\right)(X, t)-\sum_{j \in J} Q_{2}\left[B_{j}, K_{\varepsilon}(X-X(\cdot, t)) w_{0}(\cdot)\right] .
\end{gathered}
$$

THEOREM 3.2. Assume that $f \in W^{4,1}\left(\mathbf{R}^{2}\right) \cap W^{4, \infty}\left(\mathbf{R}^{2}\right)$.

(a) If there exists a constant $C_{1}>0$ such that

$$
|x|^{|\alpha|+2}\left|\partial^{\alpha} f(x)\right| \leq C_{1} \quad \text { for all }|\alpha| \leq 4 \text { and } x \in \mathbf{R}^{2}
$$

then there is a constant $C=C\left(\Omega, w_{0}, p, T\right)>0$ such that

$$
\begin{gathered}
\|\mathrm{II}(\cdot, t)\|_{L^{\infty}(\Omega)} \leq C \frac{h^{4}}{\varepsilon^{3}} \\
\|\operatorname{II}(t)\|_{h, p} \leq C \frac{h^{4}}{\varepsilon^{3}} \quad \text { for all } t \in[0, T] \text { and } 1 \leq p \leq \infty .
\end{gathered}
$$

(b) If we only assume that $|x|^{2}|f(x)| \leq C_{1}$, then there is a constant $C_{s}=$ $C_{s}\left(\Omega, w_{0}, p, T\right)>0$ such that

$$
\begin{gathered}
\|\mathrm{II}(\cdot, t)\|_{L^{\infty}(\Omega)} \leq C_{s} \frac{h^{4}}{\varepsilon^{3+s}} \\
\|\mathrm{II}(t)\|_{h, p} \leq C_{s} \frac{h^{4}}{\varepsilon^{3+s}} \quad \text { for all } t \in[0, T] \text { and } 1 \leq p \leq \infty,
\end{gathered}
$$

where $s>0$ is an arbitrary number. 
Proof. (a) By the definition, we have $|\operatorname{II}(x, t)|=\left|K_{\varepsilon} * w(x, t)-K_{\varepsilon} * w^{h}(x, t)\right|$. So

$$
\begin{aligned}
|\mathrm{II}(x, t)| & =\left|\sum_{j \in J}\left\{\int_{B_{j}} K_{\varepsilon}(x-X(y, t)) w_{0}(y) d y-Q_{2}\left[B_{j}, K_{\varepsilon}(x-X(\cdot, t)) w_{0}(\cdot)\right]\right\}\right| \\
& \leq \sum_{j \in J}\left|\left\{\int_{B_{j}} K_{\varepsilon}(x-X(y, t)) w_{0}(y) d y-Q_{2}\left[B_{j}, K_{\varepsilon}(x-X(\cdot, t)) w_{0}(\cdot)\right]\right\}\right| \\
& \leq C_{2} h^{4} \sum_{j \in J}\left|K_{\varepsilon}(x-X(\cdot, t)) w_{0}(\cdot)\right|_{4,1, B j} \quad(\text { by Lemma 3.6) } \\
& \leq C_{2} h^{4} \sum_{|\alpha|+|\beta|=4} \int_{\Omega}\left|\partial_{y}^{\alpha} K_{\varepsilon}(x-X(y, t)) \partial_{y}^{\beta} w_{0}(y)\right| d y .
\end{aligned}
$$

Using the smoothness of $u$, we have $\left|\partial_{y}^{\alpha} X_{i}(y, t)\right| \leq C_{3}$ for $i=1,2, y \in \Omega, 0 \leq|\alpha| \leq$ 4. Now using the chain rule,

$$
\begin{aligned}
|\mathrm{II}(x, t)| & \leq C_{4} h^{4} \sum_{|\alpha|+|\beta| \leq 4} \int_{\Omega}\left|\partial_{X}^{\alpha} K_{\varepsilon}(x-X(y, t)) \partial_{y}^{\beta} w_{0}(y)\right| d y \\
& \leq C_{5} h^{4} \sum_{|\alpha| \leq 4} \int_{\Omega}\left|\partial_{X}^{\alpha} K_{\varepsilon}(x-X(y, t))\right| d y \\
& =C_{5} h^{4} \sum_{|\alpha| \leq 4} \int_{\Omega}\left|\partial_{X}^{\alpha} K_{\varepsilon}(x-X)\right| d X \\
& =C_{5} h^{4} \sum_{|\alpha| \leq 4}\left\|\partial^{\alpha} K_{\varepsilon}(x-\cdot)\right\|_{L^{1}(\Omega)} \quad(\operatorname{det} J=1) .
\end{aligned}
$$

If $x \in \Omega$ and $X \in \Omega$, since $\Omega$ is bounded there exists a compact set $\mathfrak{C} \subset \mathbf{R}^{2}$, such that $x-X \in \mathfrak{C}$. By Lemma 3.4, there is a constant $C_{6}=C_{6}(\mathfrak{C})$ such that $\left\|\partial^{\alpha} K_{\varepsilon}(x-\cdot)\right\|_{L^{1}(\Omega)} \leq\left\|\partial^{\alpha} K_{\varepsilon}(\cdot)\right\|_{L^{1}(\mathfrak{C})} \leq C_{6} / \varepsilon^{3}$ for all $x \in \Omega$ and $|\alpha| \leq 4$, and so,

$$
|\mathrm{II}(x, t)|=\left|u_{\varepsilon}(x, t)-u_{\varepsilon}^{h}(x, t)\right| \leq C_{7} \frac{h^{4}}{\varepsilon^{3}}, \quad \forall x \in \Omega .
$$

This implies that

$$
\left\|u_{\varepsilon}(\cdot, t)-u_{\varepsilon}^{h}(\cdot, t)\right\|_{L^{\infty}(\Omega)} \leq C_{7} \frac{h^{4}}{\varepsilon^{3}} .
$$

For $1 \leq p<\infty$, it follows that

$$
\begin{aligned}
\|\mathrm{II}(t)\|_{h, p} & =\left\{\sum_{i \in I} h^{2}\left|u_{\varepsilon}\left(X\left(x_{i}, t\right), t\right)-u_{\varepsilon}^{h}\left(X\left(x_{i}, t\right), t\right)\right|^{p}\right\}^{1 / p} \\
& \leq\left\|u_{\varepsilon}(\cdot, t)-u_{\varepsilon}^{h}(\cdot, t)\right\|_{L^{\infty}(\Omega)}\left\{\sum_{i \in I} h^{2}\right\}^{1 / p} \\
& \leq C_{8}(\text { measure } \Omega)^{1 / p} h^{4} / \varepsilon^{3}=C_{9} h^{4} / \varepsilon^{3}
\end{aligned}
$$

(b) As in (a), we have

$$
|\mathrm{II}(x, t)| \leq C_{4} h^{4} \sum_{|\alpha|+|\beta| \leq 4} \int_{\Omega}\left|\partial_{X}^{\alpha} K_{\varepsilon}(x-X(y, t)) \partial_{y}^{\beta} w_{0}(y)\right| d y .
$$


This time, Lemma 3.4 is not directly available to estimate $\left\|\partial^{\alpha} K_{\varepsilon}(x-\cdot)\right\|_{L^{1}(\Omega)}$, for all $|\alpha| \leq 4$. For $|\alpha|=0$ and $|\beta| \leq 4$, by Lemma 3.4 as above,

$$
\left\|\partial_{X}^{\alpha} K_{\varepsilon}(x-X(\cdot, t)) \partial_{y}^{\beta} w_{0}(\cdot)\right\|_{L^{1}(\Omega)} \leq C_{10}\left\|K_{\varepsilon}(x-X(\cdot, t))\right\|_{L^{1}(\Omega)} \leq C_{11}(\mathfrak{C}) .
$$

For $|\alpha| \geq 1$ and $|\beta| \leq 3$, using Hölder's inequality, we obtain

$$
\begin{aligned}
& \left\|\partial_{X}^{\alpha} K_{\varepsilon}(x-X(\cdot, t)) \partial_{y}^{\beta} w_{0}(\cdot)\right\|_{L^{1}(\Omega)} \\
& \quad \leq\left\|\partial_{X}^{\alpha} K_{\varepsilon}(x-X(\cdot, t))\right\|_{L^{p^{\prime}}(\Omega)}\left\|\partial_{y}^{\beta} w_{0}(\cdot)\right\|_{L^{q^{\prime}}(\Omega)} \\
& \quad\left(1 / p^{\prime}+1 / q^{\prime}=1 \text { and } 1<p^{\prime}<\infty\right) \\
& \quad \leq C_{12}\left\|\partial_{X}^{\alpha} K_{\varepsilon}(x-X(\cdot, t))\right\|_{L^{p^{\prime}}(\Omega)} \leq C_{12}\left\|\partial_{X}^{\alpha} K_{\varepsilon}(\cdot)\right\|_{L^{p^{\prime}}\left(\mathbf{R}^{2}\right)}
\end{aligned}
$$

Using Lemma 3.2,

$$
\left\|\partial_{X}^{\alpha} K_{\varepsilon}(\cdot)\right\|_{L^{p^{\prime}}\left(\mathbf{R}^{2}\right)} \leq \frac{C_{12}}{\varepsilon^{|\alpha|-1+2 / q^{\prime}}} \leq \frac{C_{12}}{\varepsilon^{3+2 / q^{\prime}}}
$$

Hence, $|\operatorname{II}(x, t)|=\left|u_{\varepsilon}(x, t)-u_{\varepsilon}^{h}(x, t)\right| \leq C_{13} h^{4} / \varepsilon^{3+s}$ for all $x \in \Omega$, where $s=2 / q^{\prime}>$ 0 . Then (b) follows.

The Stability Error. By definition, this is

$$
u_{\varepsilon}^{h}(X, t)-\mathscr{U}_{\varepsilon}^{h}\left(X^{h}, t\right)=\left[u_{\varepsilon}^{h}(X, t)-\mathscr{U}_{\varepsilon}^{h}(X, t)\right]+\left[\mathscr{U}_{\varepsilon}^{h}(X, t)-\mathscr{U}_{\varepsilon}^{h}\left(X^{h}, t\right)\right],
$$

where $X=X(x, t)$ and $X^{h}=X^{h}(x, t)$ for $x \in \Omega$. We call the expression in the first bracket Part I, the other Part II.

Part I. By Table 1,

$$
\begin{aligned}
u_{\varepsilon}^{h}(X, & t)-\mathscr{U}_{\varepsilon}^{h}(X, t)=K_{\varepsilon} * w^{h}(X, t)-K_{\varepsilon} * w^{h}(X, t) \\
= & \sum_{i \in I}\left[c_{i} K_{\varepsilon}\left(X-X\left(x_{i}, t\right)\right)-d_{1 i}(t) K_{\varepsilon x_{1}}\left(X-X\left(x_{i}, t\right)\right)\right. \\
& -\sum_{i \in I}\left[c_{i} K_{\varepsilon}\left(X-X^{h}\left(x_{i}, t\right)\right)-d_{1 i}(t) K_{\varepsilon x_{1}}\left(X-X^{h}\left(x_{i}, t\right)\right)\right. \\
= & \left\{\sum _ { i \in I } c _ { i } \left[K_{\varepsilon}\left(X-d_{2 i}(t) K_{\varepsilon x_{2}}\left(X-X^{h}\left(x_{i}, t\right)\right)\right]\right.\right. \\
+ & \left.\left\{-\sum_{i \in I} d_{1 i}(t)\left[K_{\varepsilon x_{1}}(X-X)\right)-K_{\varepsilon}\left(X-X^{h}\left(x_{i}, t\right)\right)\right]\right\} \\
+ & \left.\left\{-\sum_{i \in I} d_{2 i}(t)\left[K_{\varepsilon x_{2}}(X-X)\right)-K_{\varepsilon x_{1}}\left(X-X^{h}\left(x_{i}, t\right)\right)\right]\right\} \\
+ & \left.\left.\left\{-\sum_{i \in I} K_{\varepsilon x_{1}}\left(X-X_{i}, t\right)\right)-K_{\varepsilon x_{2}}\left(X-X^{h}\left(x_{i}, t\right)\right)\right]\right\} \\
+ & \left\{-\sum_{i \in I} K_{\varepsilon x_{2}}\left(X-X^{h}\left(x_{i}, t\right)\right)\left[d_{2 i}(t)-d_{2 i}(t)\right]\right\} \\
= & V_{11}(X, t)+V_{12}(X, t)+V_{13}(X, t)+V_{21}(X, t)+V_{22}(X, t) .
\end{aligned}
$$


Here, for $i=1,2, j=1,2$ (or 1,2 , and 3 ), we use $V_{i j}(X, t)$ to denote the terms in each pair of braces in the above equation, respectively. Since

$$
K_{\varepsilon x_{1}}=\partial\left(K * f_{\varepsilon}\right) / \partial x_{1}=K *\left(\partial f_{\varepsilon} / \partial x_{1}\right)
$$

and

$$
\partial f_{\varepsilon} / \partial x_{1}=\frac{\partial}{\partial x_{1}}\left[f(x / \varepsilon) / \varepsilon^{2}\right]=f_{x_{1}}(x / \varepsilon) / \varepsilon^{3}
$$

where $f_{x_{1}}=\partial f(x) / \partial x_{1}$,

$$
K_{\varepsilon x_{1}}=\left(K * f_{x_{1} \varepsilon}\right) / \varepsilon
$$

Similarly,

$$
K_{\varepsilon x_{2}}=\left(K * f_{x_{2} \varepsilon}\right) / \varepsilon \text {. }
$$

Concerning terms $V_{1 j}(X, t), j=1,2$ and 3 , we have

$$
\begin{aligned}
& V_{11}(X, t)=\left.\sum_{i \in I} c_{i} \mid K_{\varepsilon}\left(X-X\left(x_{i}, t\right)\right)-K_{\varepsilon}\left(X-X^{h}\left(x_{i}, t\right)\right)\right] \quad(\text { by }(2.4)) \\
&= \sum_{i \in I}\left|S_{i}\right|\left(w_{0}\left(x_{i}\right)+H_{i 1} w_{0 x_{1}}\left(x_{i}\right)+H_{i 2} w_{0 x_{2}}\left(x_{i}\right)\right) \\
& \cdot\left[K_{\varepsilon}\left(X-X\left(x_{i}, t\right)\right)-K_{\varepsilon}\left(X-X^{h}\left(x_{i}, t\right)\right)\right] \\
& V_{12}(X, t)=-\sum_{i \in I} d_{1 i}(t)\left[K_{\varepsilon x_{1}}\left(X-X\left(x_{i}, t\right)\right)-K_{\varepsilon x_{1}}\left(X-X^{h}\left(x_{i}, t\right)\right)\right] \\
&=\sum_{i \in I}\left|S_{i}\right|\left\{M_{11}\left(x_{i}, t\right) H_{i 1} / \varepsilon+M_{12}\left(x_{i}, t\right) H_{i 2} / \varepsilon\right\} w_{0}\left(x_{i}\right) \\
& \cdot\left[K * f_{x_{1} \varepsilon}\left(X-X\left(x_{i}, t\right)\right)-K * f_{x_{1} \varepsilon}\left(X-X^{h}\left(x_{i}, t\right)\right)\right] .
\end{aligned}
$$

Similarly,

$$
\begin{aligned}
& V_{13}(X, t)=-\sum_{i \in I} d_{2 i}(t)\left[K_{\varepsilon x_{2}}\left(X-X\left(x_{i}, t\right)\right)-K_{\varepsilon x_{2}}\left(X-X^{h}\left(x_{i}, t\right)\right)\right] \\
&= \sum_{i \in I}\left|S_{i}\right|\left\{M_{21}\left(x_{i}, t\right) H_{i 1} / \varepsilon+M_{22}\left(x_{i}, t\right) H_{i 2} / \varepsilon\right\} w_{0}\left(x_{i}\right) \\
& \cdot\left[K * f_{x_{2} \varepsilon}\left(X-X\left(x_{i}, t\right)\right)-K * f_{x_{2} \varepsilon}\left(X-X^{h}\left(x_{i}, t\right)\right)\right] .
\end{aligned}
$$

Define

$$
V_{1}(X, t)=\sum_{i \in I}\left|S_{i}\right| \alpha_{i}\left\{K * g_{\varepsilon}\left(X-X\left(x_{i}, t\right)\right)-K * g_{\varepsilon}\left(X-X^{h}\left(x_{i}, t\right)\right)\right\},
$$

where $\left\{\alpha_{i}\right\}_{i \in I}$ is a family of real numbers, $g(\cdot)$ is a cutoff function which can be $f(\cdot)$ or the partial derivatives of $f(\cdot)$, and $g_{\varepsilon}(x)=g(x / \varepsilon) / \varepsilon^{2}$.

LEMMA 3.7. Assume the conditions:

(1) $g(\cdot) \in W^{1, \infty}\left(\mathbf{R}^{2}\right)$ and there are two constants $C_{1}>0$ and $\gamma>2$ such that

$$
\left|\partial^{\alpha} g(x)\right| \leq C_{1}(1+|x|)^{-\gamma} \quad \forall x \in \mathbf{R}^{2},|\alpha|=0,1 .
$$

(2) There is a constant $C_{2}>0$ such that $h / \varepsilon \leq C_{2}$.

(3) There is a constant $C_{3}>0, C_{3}=C_{3}\left(w_{0}, T\right)$, such that $\left|\alpha_{i}\right| \leq C_{3}$ for all $i \in I$. 
Then, for $p \in(1, \infty)$, there exists a constant $C=C\left(p, T, w_{0}\right)$ such that

$$
\left\|V_{1}(\cdot, t)\right\|_{L^{p}\left(\mathbf{R}^{2}\right)}+\varepsilon\left|V_{1}(\cdot, t)\right|_{1, p, \mathbf{R}^{2}} \leq C\left(1+\|e(t)\|_{h, \infty} / \varepsilon\right)^{2 / q}\|e(t)\|_{h, p},
$$

where $1 / p+1 / q=1$ and the discrete norms are defined in Definition 3.1.

Proof. [18, Chapter II, Lemma 5.2 and Lemma 5.3: Substitute $C_{3}$ in (3) for $\left.\left\|w_{0}\right\|_{L^{\infty}\left(\mathbf{R}^{2}\right)}\right]$.

Remark. Although Lemma 5.2 and Lemma 5.3 in [18] are proved for uniform meshes, the generalization to the nonuniform case is straightforward. Several similar direct extensions are used below without comment.

In order to find a bound for the discrete norm of $V_{1}$, we need the following standard result in finite element theory.

LEMMA 3.8. For all $p>2$ and all functions $g \in W^{1, p}\left(\mathbf{R}^{2}\right)$,

$$
\|g(t)\|_{h, p}=\left\{h^{2} \sum_{i \in Z^{n}} \mid g\left(\left.X\left(x_{i}, t\right)\right|^{p}\right\}^{1 / p} \leq C\left\{\|g\|_{L^{p}\left(\mathbf{R}^{2}\right)}+h|g|_{1, p, \mathbf{R}^{2}}\right\} .\right.
$$

Proof. See [18, Chapter II, Lemma 5.4].

COROllary 3.1. Assume conditions (1), (2) and (3) in Lemma 3.7; then, for $2<p<\infty$, there exists a constant $C=C\left(p, T, w_{0}\right)$ such that

$$
\left\|V_{1}(t)\right\|_{h, p} \leq C\left(1+\|e(t)\|_{h, \infty} / \varepsilon\right)^{2 / q}\|e(t)\|_{h, p} .
$$

Proof. The proof follows directly from Lemma 3.7 and Lemma 3.8.

THEOREM 3.3. Assume the conditions:

(1) $f(\cdot) \in W^{2, \infty}\left(\mathbf{R}^{2}\right)$ and there are two constants $C_{1}>0$ and $\gamma>2$ such that

$$
\left|\partial^{\alpha} f(x)\right| \leq C_{1}(1+|x|)^{-\gamma} \quad \forall x \in \mathbf{R}^{2},|\alpha|=0,1,2 .
$$

(2) There is a constant $C_{2}>0$ such that $h / \varepsilon \leq C_{2}$.

Then, for $2<p<\infty$, there exists a constant $C=C\left(p, T, w_{0}\right)$ such that

$$
\left\|V_{11}(t)\right\|_{h, p}+\left\|V_{12}(t)\right\|_{h, p}+\left\|V_{13}(t)\right\|_{h, p} \leq C\left(1+\|e(t)\|_{h, \infty} / \varepsilon\right)^{2 / q}\|e(t)\|_{h, p},
$$

where $1 / p+1 / q=1$.

Proof. Since the solution of the Euler equation is assumed to be smooth for $t \in[0, T], M(x, t), w_{0}(x)$ and $\nabla w_{0}(x)$ are uniformly bounded for all $x \in \Omega$. So,

$$
\begin{gathered}
\left\{w_{0}\left(x_{i}\right)+H_{i 1} w_{0 x_{1}}\left(x_{i}\right)+H_{i 2} w_{0 x_{2}}\left(x_{i}\right)\right\}_{i \in I}, \\
\left\{\left[M_{11}\left(x_{i}, t\right) H_{i 1} / \varepsilon+M_{12}\left(x_{i}, t\right) H_{i 2} / \varepsilon\right] w_{0}\left(x_{i}\right)\right\}_{i \in I} \text { and } \\
\left\{\left[M_{21}\left(x_{i}, t\right) H_{i 1} / \varepsilon+M_{22}\left(x_{i}, t\right) H_{i 2} / \varepsilon\right] w_{0}\left(x_{i}\right)\right\}_{i \in I}
\end{gathered}
$$

are all uniformly bounded by some constant which is independent of $h$ and $\varepsilon$. Thus, Theorem 3.3 follows from Lemma 3.7, Lemma 3.8 and Corollary 3.1.

For estimating $V_{21}$ and $V_{22}$, the following lemmas are needed. 
LEMMA 3.9. Assume the following conditions:

(1) $g(\cdot) \in W^{\ell, 1}\left(\mathbf{R}^{2}\right) \cap W^{\ell, \infty}\left(\mathbf{R}^{2}\right)$ and there is a constant $C_{1}>0$ such that for $|\beta|=\ell,|x|^{\ell+2}\left|\partial^{\beta} g(x)\right| \leq C_{1}$ for all $x \in \mathbf{R}^{2}$.

(2) There is a constant $C_{2}$ such that $h / \varepsilon \leq C_{2}$.

Then, for any compact set $\mathscr{S} \in \mathbf{R}^{2}$, there exists a constant $C=C(\mathscr{S})$ such that

$$
\sum_{i \in I,\left|y_{i}\right| \leq\|e(t)\|_{h, \infty}}\left|\partial^{\beta} K * g_{\varepsilon}\left(x-X\left(x_{i}, t\right)+y_{i}\right)\right| h^{2} \leq B(\beta, \varepsilon)
$$

for all $x \in \mathscr{S}, t \in\left[0, T_{\varepsilon}\right]$ and $|\beta|=\ell$, where $T_{\varepsilon}=\max \left\{t \in[0, T] \mid\|e(t)\|_{h, \infty} \leq M \varepsilon\right.$; $M$ is an arbitrary constant $\}$ and

$$
B(\beta, \varepsilon)= \begin{cases}C, & |\beta|=0 \\ C|\log \varepsilon|, & |\beta|=1, \\ C \varepsilon^{1-|\beta|}, & |\beta|>1\end{cases}
$$

Proof. For any $i \in I$, as in the proof of Lemma 5.2 in [18], the area of $S_{i}(t)$ is of order $h^{2}$. Let $a=\max _{i \in I} \max _{y \in S_{i}}\left|X(y, t)-X^{h}\left(x_{i}, t\right)\right|$; then

$$
\|e(t)\|_{h, \infty} \leq a \leq C_{3} h+\|e(t)\|_{h, \infty} .
$$

For a fixed $x \in \mathscr{S}$, let $J_{1}=\left\{i \in I|| x-X\left(x_{i}, t\right) \mid \leq \varepsilon+a\right\}$. If $i \in J_{1}$, then $\left|x-X\left(x_{i}, t\right)\right| \leq \varepsilon+a \leq \varepsilon+C_{3} h+\|e(t)\|_{h, \infty}$. So, $X\left(x_{i}, t\right) \in S\left(x, \varepsilon+C_{3} h+\|e(t)\|_{h, \infty}\right)$. This implies that Card $J_{1} \leq C_{4}\left(\varepsilon / h+1+\|e(t)\|_{h, \infty} / h\right)^{2}$. So,

$$
\begin{aligned}
& \sum_{i \in J_{1},\left|y_{i}\right| \leq\|e(t)\|_{h, \infty}}\left|\partial^{\beta} K * g_{\varepsilon}\left(x-X\left(x_{i}, t\right)+y_{i}\right)\right| h^{2} \leq \text { Card } J_{1} h^{2}\left\|\partial^{\beta} K * g_{\varepsilon}\right\|_{L^{\infty}\left(\mathbf{R}^{2}\right)} \\
\leq & C_{4} \frac{\left(\varepsilon / h+1+\|e(t)\|_{h, \infty} / h\right)^{2} h^{2}}{\varepsilon^{\ell+1}} \leq C_{5}\left(1+\|e(t)\|_{h, \infty} / \varepsilon\right)^{2} \cdot \varepsilon^{1-\ell} \\
\leq & C_{6} \varepsilon^{1-\ell} \quad \text { for all } t \in\left[0, T_{\varepsilon}\right](\text { Lemma } 3.3(\mathrm{a})) .
\end{aligned}
$$

Let $J_{2}=I \backslash J_{1}$. If $i \in J_{2}$, then $\left|x-X\left(x_{i}, t\right)\right| \geq \varepsilon+a$. So, $\left|x-X\left(x_{i}, t\right)+y_{i}\right| \geq$ $\varepsilon+a-\|e(t)\|_{h, \infty} \geq \varepsilon$, because of (3.8). Using Lemma 3.3(b), we obtain

$$
\left|\partial^{\beta} K * g_{\varepsilon}\left(x-X\left(x_{i}, t\right)+y_{i}\right)\right| \leq \frac{C_{7}}{\left|x-X\left(x_{i}, t\right)+y_{i}\right|^{\ell+1}} \leq \frac{C_{7}}{\left\{\left|x-X\left(x_{i}, t\right)\right|-a\right\}^{\ell+1}},
$$

so that,

$\sum_{i \in J_{2},\left|y_{i}\right| \leq\|e(t)\|_{h, \infty}}\left|\partial^{\beta} K * g_{\varepsilon}\left(x-X\left(x_{i}, t\right)+y_{i}\right)\right| h^{2} \leq C_{7} \sum_{i \in J_{2}} \frac{h^{2}}{\left\{\left|x-X\left(x_{i}, t\right)\right|-a\right\}^{\ell+1}}$.

When $h \rightarrow 0$,

$$
\lim _{h \rightarrow 0} \sum_{i \in J_{2}} \frac{h^{2}}{\left\{\left|x-X\left(x_{i}, t\right)\right|-a\right\}^{\ell+1}} \leq \int_{\substack{|x-y| \geq \varepsilon+a \\ y \in \Omega}} \frac{d y}{\{|x-y|-a\}^{\ell+1}} .
$$

Since $x \in \mathscr{S}, y \in \Omega$, where $\mathscr{S}$ is compact and $\Omega$ is bounded, there exists a constant $R>0$ such that $|x-y| \leq R$, and therefore,

$$
\begin{aligned}
\int_{\substack{|x-y| \geq \varepsilon+a \\
y \in \Omega}} \frac{d y}{\{|x-y|-a\}^{\ell+1}} & \leq \int_{0}^{2 \pi} \int_{\varepsilon+a \leq r \leq R} \frac{r d r d \theta}{(r-a)^{\ell+1}} \\
& =\int_{0}^{2 \pi} \int_{\varepsilon}^{R-a} \frac{(r+a) d r d \theta}{r^{\ell+1}}
\end{aligned}
$$


Since $a \leq C_{3} h+\|e(t)\|_{h, \infty} \leq C_{3} h+M \varepsilon \leq C_{8} \varepsilon \leq C_{8} r$ for all $t \in\left[0, T_{\varepsilon}\right]$, it follows that

$$
\begin{aligned}
\int_{0}^{2 \pi} \int_{\varepsilon}^{R-a} \frac{(r+a) d r d \theta}{r^{\ell+1}} \leq\left(1+C_{8}\right) 2 \pi \int_{\varepsilon \leq r \leq R-a} \frac{d r}{r^{\ell}} & |\beta|=0 \\
= & \begin{cases}\left(1+C_{8}\right) 2 \pi(R-a-\varepsilon), & |\beta|=1 \\
\left(1+C_{8}\right) 2 \pi(\log (R-a)-\log \varepsilon), & 1 \\
\left(1+C_{8}\right) 2 \pi \frac{1}{\ell-1}\left[\frac{1}{\varepsilon^{\ell-1}}-\frac{1}{(R-a)^{\ell-1}}\right], & |\beta|>1\end{cases}
\end{aligned}
$$

Define

$$
V_{2}(X, t)=\sum_{i \in I}\left|S_{i}\right| \alpha_{i}\left\{\partial^{\beta} K * g_{\varepsilon}\left(X-X^{h}\left(x_{i}, t\right)\right)\right\}
$$

where $\left\{\alpha_{i}\right\}_{i \in I}$ and $g(\cdot)$ are the same as in (3.5). Then we have

LEMMA 3.10. Assume the conditions (1) and (2) in Lemma 3.9. Then there exists a constant $C=C(\Omega, p)$ such that

$$
\left\|V_{2}(t)\right\|_{h, p} \leq B(\beta, \varepsilon)\left\{\sum_{i \in I}\left|\alpha_{i}\right|^{p} h^{2}\right\}^{1 / p} \quad \forall p \in(1, \infty) \text { and } t \in\left[0, T_{\varepsilon}\right],
$$

where $T_{\varepsilon}$ and $B(\beta, \varepsilon)$ are the same as in Lemma 3.9 .

Proof. For $j \in I$ and $i \in I$,

$$
X\left(x_{j}, t\right)-X^{h}\left(x_{i}, t\right)=\left[X\left(x_{j}, t\right)-X\left(x_{i}, t\right)\right]+\left[X\left(x_{i}, t\right)-X^{h}\left(x_{i}, t\right)\right] .
$$

Let $y_{i}=X\left(x_{i}, t\right)-X^{h}\left(x_{i}, t\right)$; then $\left|y_{i}\right| \leq\|e(t)\|_{h, \infty}$. So, Lemma 3.9 implies that for $t \in\left[0, T_{\varepsilon}\right]$,

$$
\sum_{i \in I}\left|\partial^{\beta} K * g_{\varepsilon}\left(X\left(x_{j}, t\right)-X^{h}\left(x_{i}, t\right)\right)\right| h^{2} \leq B_{1}(\beta, \varepsilon) .
$$

Let $g(x)=g(-x)$. Since $K(y)=-K(-y)$,

$$
K * g_{\varepsilon}(x)=\int_{\mathbf{R}^{2}} K(y) g_{\varepsilon}(x-y) d y=\int_{\mathbf{R}^{2}} K(y) g_{\varepsilon}(-x-y) d y=K * g_{\varepsilon}(-x) .
$$

So we have

$$
K * g_{\varepsilon}\left(X\left(x_{j}, t\right)-X^{h}\left(x_{i}, t\right)\right)=K * g_{\varepsilon}\left(X^{h}\left(x_{i}, t\right)-X\left(x_{j}, t\right)\right) .
$$

Also,

$$
X^{h}\left(x_{i}, t\right)-X\left(x_{j}, t\right)=\left[X\left(x_{i}, t\right)-X\left(x_{j}, t\right)\right]+\left[X^{h}\left(x_{i}, t\right)-X\left(x_{i}, t\right)\right],
$$

and letting $y_{i}=X^{h}\left(x_{i}, t\right)-X\left(x_{i}, t\right)$, we have $\left|y_{i}\right| \leq\|e(t)\|_{h, \infty}$. Substitute $g$ for $g$ in Lemma 3.9; then, for $t \in\left[0, T_{\varepsilon}\right]$, we have

$$
\begin{aligned}
& \sum_{j \in I}\left|\partial^{\beta} K * g_{\varepsilon}\left(X\left(x_{j}, t\right)-X^{h}\left(x_{i}, t\right)\right)\right| h^{2} \\
& =\sum_{j \in I}\left|\partial^{\beta} K * g_{\varepsilon}\left(X^{h}\left(x_{i}, t\right)-X\left(x_{j}, t\right)\right)\right| h^{2} \leq B_{2}(\beta, \varepsilon),
\end{aligned}
$$




$$
\begin{aligned}
\left|V_{2}\left(X\left(x_{j}, t\right), t\right)\right|=\left|\sum_{i \in I}\right| S_{i}\left|\alpha_{i}\left\{\partial^{\beta} K * g_{\varepsilon}\left(X\left(x_{j}, t\right)-X^{h}\left(x_{i}, t\right)\right)\right\}\right| \\
\leq C_{3} \sum_{i \in I}\left|\alpha_{i}\right|\left|\partial^{\beta} K * g_{\varepsilon}\left(X\left(x_{j}, t\right)-X^{h}\left(x_{i}, t\right)\right)\right|^{1 / p+1 / q} h^{2 / p+2 / q} \\
\leq C_{3}\left\{\sum_{i \in I}\left|\partial^{\beta} K * g_{\varepsilon}\left(X\left(x_{j}, t\right)-X^{h}\left(x_{i}, t\right)\right)\right| h^{2}\right\}^{1 / q} \\
\\
\quad\left\{\sum_{i \in I}\left|\alpha_{i}\right|^{p}\left|\partial^{\beta} K * g_{\varepsilon}\left(X\left(x_{j}, t\right)-X^{h}\left(x_{i}, t\right)\right)\right| h^{2}\right\}^{1 / p}(\text { Hölder Inequality) } \\
\leq C_{3}\left(B_{1}(\beta, \varepsilon)\right)^{1 / q}\left\{\sum_{i \in I}\left|\alpha_{i}\right|^{p}\left|\partial^{\beta} K * g_{\varepsilon}\left(X\left(x_{j}, t\right)-X^{h}\left(x_{i}, t\right)\right)\right| h^{2}\right\}^{1 / p} .
\end{aligned}
$$

From this, we obtain

$$
\begin{aligned}
& \left|V_{2}\left(X\left(x_{j}, t\right), t\right)\right|^{p} \\
& \quad \leq\left(C_{3}\right)^{p}\left(B_{1}(\beta, \varepsilon)\right)^{p / q}\left\{\sum_{i \in I}\left|\alpha_{i}\right|^{p}\left|\partial^{\beta} K * g_{\varepsilon}\left(X\left(x_{j}, t\right)-X^{h}\left(x_{i}, t\right)\right)\right| h^{2}\right\}
\end{aligned}
$$

and

$$
\begin{aligned}
& \left\|V_{2}(t)\right\|_{h, p}^{p}=\sum_{j \in I}\left|V_{2}\left(X\left(x_{j}, t\right), t\right)\right|^{p} h^{2} \\
& \leq\left(C_{3}\right)^{p}\left(B_{1}(\beta, \varepsilon)\right)^{p / q}\left\{\sum_{j \in I}\left[\sum_{i \in I}\left|\alpha_{i}\right|^{p}\left|\partial^{\beta} K * g_{\varepsilon}\left(X\left(x_{j}, t\right)-X^{h}\left(x_{i}, t\right)\right)\right| h^{2}\right] h^{2}\right\} \\
& =\left(C_{3}\right)^{p}\left(B_{1}(\beta, \varepsilon)\right)^{p / q}\left\{\sum_{i \in I}\left[\sum_{j \in I}\left|\partial^{\beta} K * g_{\varepsilon}\left(X\left(x_{j}, t\right)-X^{h}\left(x_{i}, t\right)\right)\right| h^{2}\right]\left|\alpha_{i}\right|^{p} h^{2}\right\} \\
& =\left(C_{3}\right)^{p}\left(B_{1}(\beta, \varepsilon)\right)^{p / q} B_{2}(\beta, \varepsilon)\left\{\sum_{i \in I}\left|\alpha_{i}\right|^{p} h^{2}\right\} .
\end{aligned}
$$

Thus,

$\left\|V_{2}(t)\right\|_{h, p} \leq C_{3}\left(B_{1}(\beta, \varepsilon)\right)^{1 / q}\left(B_{2}(\beta, \varepsilon)\right)^{1 / p}\left\{\sum_{i \in I}\left|\alpha_{i}\right|^{p} h^{2}\right\}^{1 / p} \quad$ for all $t \in\left[0, T_{\varepsilon}\right]$

THEOREM 3.4. Assume the following conditions:

(1) $f(\cdot) \in W^{1,1}\left(\mathbf{R}^{2}\right) \cap W^{1, \infty}\left(\mathbf{R}^{2}\right)$. There is a constant $C_{1}>0$ such that $|x|^{2}\left|\partial^{\alpha} f(x)\right| \leq C_{1}$ for all $x \in \mathbf{R}^{2},|\alpha|=1$.

(2) There is a constant $C_{2}$ such that $h \leq C_{2} \varepsilon^{2}$.

Then there exists a constant $C=C\left(\Omega, p, w_{0}\right)$ such that

$$
\left\|V_{21}(t)\right\|_{h, p}+\left\|V_{22}(t)\right\|_{h, p} \leq C \varepsilon\|E(t)\|_{h, p} \quad \forall t \in\left[0, T_{\varepsilon}\right] .
$$

Proof. By definition,

$$
\begin{aligned}
V_{21}(X, t) & =-\sum_{i \in I} K_{\varepsilon x_{1}}\left(X-X^{h}\left(x_{i}, t\right)\right)\left[d_{1 i}(t)-d_{1 i}(t)\right] \\
& =-(1 / \varepsilon) \sum_{i \in I} K * f_{x_{1} \varepsilon}\left(X-X^{h}\left(x_{i}, t\right)\right)\left[d_{1 i}(t)-d_{1 i}(t)\right]
\end{aligned}
$$


By Lemma 3.10 with $\beta=0$ and $g=f_{x_{1} \varepsilon}$, there is a constant $C_{3}=C_{3}(\Omega, p)$ such that

$$
\left\|V_{21}(t)\right\|_{h, p} \leq\left(C_{3} / \varepsilon\right)\left\{\sum_{i \in I}\left\|\left[d_{1 i}(t)-d_{1 i}(t)\right] / \mid S_{i}\right\|^{p} h^{2}\right\}^{1 / p} .
$$

According to the construction of the method in Section 2,

$$
\begin{aligned}
{\left[d_{1 i}(t)\right.} & \left.-d_{1 i}(t)\right] /\left|S_{i}\right| \\
= & {\left[M_{11}\left(x_{i}, t\right) d_{1 i}+M_{12}\left(x_{i}, t\right) d_{2 i}-M_{11}^{h}\left(x_{i}, t\right) d_{1 i}-M_{12}^{h}\left(x_{i}, t\right) d_{2 i}\right] /\left|S_{i}\right| } \\
= & {\left[M_{11}\left(x_{i}, t\right)-M_{11}^{h}\left(x_{i}, t\right)\right]\left(-H_{i 1} w_{0}\left(x_{i}\right)\right) } \\
& +\left[M_{12}\left(x_{i}, t\right)-M_{12}^{h}\left(x_{i}, t\right)\right]\left(-H_{i 2} w_{0}\left(x_{i}\right)\right),
\end{aligned}
$$

so that

$$
\left|d_{1 i}(t)-d_{1 i}(t)\right| /\left|S_{i}\right| \leq 2 h\left\|w_{0}\right\|_{L^{\infty}\left(\mathbf{R}^{2}\right)}\left|M\left(x_{i}, t\right)-M^{h}\left(x_{i}, t\right)\right| .
$$

Hence, $\left\|V_{21}(t)\right\|_{h, p} \leq\left(C_{4} h / \varepsilon\right)\|E(t)\|_{h, p} \leq\left(C_{4} C_{2} \varepsilon\right)\|E(t)\|_{h, p}$ (condition (2)), and in a similar way, $\left\|V_{22}(t)\right\|_{h, p} \leq\left(C_{5} C_{2} \varepsilon\right)\|E(t)\|_{h, p}$.

Part II. Consider the difference

$$
\begin{aligned}
\mathscr{U}_{\varepsilon}^{h}(X, t)-\mathscr{U}_{\varepsilon}^{h}\left(X^{h}, t\right)=K_{\varepsilon} * \omega^{h}(X, t)-K_{\varepsilon} * \omega^{h}\left(X^{h}, t\right) & \left.-d_{2 i}(t) K_{\varepsilon x_{2}}\left(X-X^{h}\left(x_{i}, t\right)\right)\right] \\
= & \sum_{i \in I}\left[c_{i} K_{\varepsilon}\left(X-X^{h}\left(x_{i}, t\right)\right)-d_{1 i}(t) K_{\varepsilon x_{1}}\left(X-X^{h}\left(x_{i}, t\right)\right)\right. \\
& -\sum_{i \in I}\left[c_{i} K_{\varepsilon}\left(X^{h}-X^{h}\left(x_{i}, t\right)\right)-d_{1 i}(t) K_{\varepsilon x_{1}}\left(X^{h}-X^{h}\left(x_{i}, t\right)\right)\right. \\
= & \left\{\sum _ { i \in I } c _ { i } \left[K_{\varepsilon}\left(X-X_{\varepsilon x_{2}}\left(X^{h}-X^{h}\left(x_{i}, t\right)\right)\right]\right.\right. \\
+ & \left\{-\sum_{i \in I} d_{1 i}(t)\left[K_{\varepsilon x_{1}}\left(X-X^{h}\right)-K_{\varepsilon}\left(X^{h}-X^{h}\left(x_{i}, t\right)\right)\right]\right\} \\
+ & \left\{-\sum_{i \in I} d_{2 i}(t)\left[K_{\varepsilon x_{2}}\left(X-X^{h}\left(x_{i}, t\right)\right)-K_{\varepsilon x_{1}}\left(X^{h}-X^{h}\left(x_{i}, t\right)\right)\right]\right\}
\end{aligned}
$$

where we call the expression in the first pair of braces $W_{11}\left(X, X^{h}, t\right)$ and the other two $W_{21}\left(X, X^{h}, t\right)$ and $W_{22}\left(X, X^{h}, t\right)$. Now we define

$$
W\left(X, X^{h}, t\right)=\sum_{i \in I}\left|S_{i}\right| \alpha_{i}\left[K * g_{\varepsilon}\left(X-X^{h}\left(x_{i}, t\right)\right)-K * g_{\varepsilon}\left(X^{h}-X^{h}\left(x_{i}, t\right)\right)\right]
$$

where $\left\{\alpha_{i}\right\}_{i \in I}, g$ and $g_{\varepsilon}$ are as before. For $\left\{\alpha_{i}\right\}_{i \in I}$, we have the following lemma, where the region $\Omega$ and $\mathscr{A}$ are as shown in Figure $5 . \mathscr{A}$ is a compact set which contains $\Omega$ and is bounded by mesh lines, and $x_{i}, x_{k}$ are adjacent mesh points in $\mathscr{A}$.

LEMMA 3.12. Consider the family of numbers $\left\{\alpha_{i} \mid i \in I, \alpha_{i}=0\right.$ if $\left.x_{i} \notin \Omega\right\}$. Assume the conditions:

(1) There exists a constant $C_{1}$ such that $\max _{j \in J}\left(h_{j 1}, h_{j 2}\right) / \min _{j \in J}\left(h_{j 1}, h_{j 2}\right) \leq$ $C_{1}$ and $h \leq 1$. 

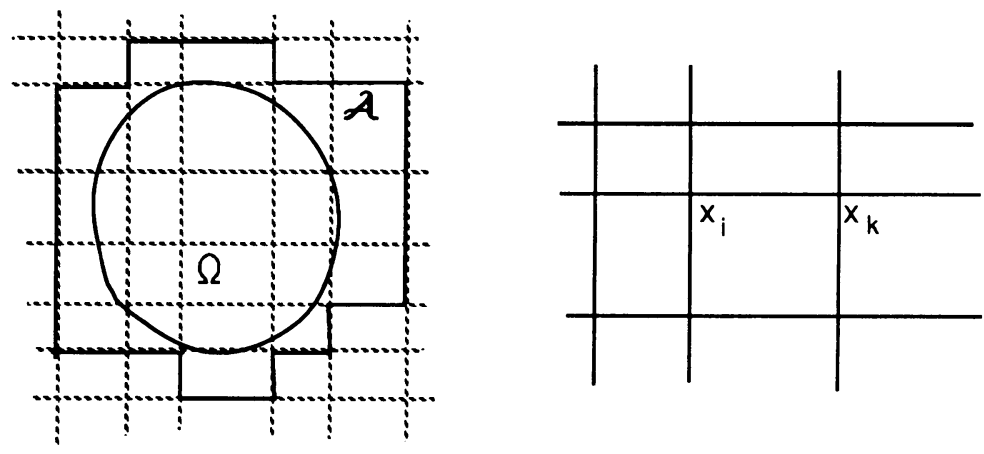

\section{FIGURE 5}

(2) There exists a constant $C_{2}$ which is independent of $h$ such that $\left|\alpha_{i}\right| \leq C_{2}$ for all $i \in I$.

(3) For any adjacent $x_{i}$ and $x_{k}$ with $i, k \in I,\left|\alpha_{i}-\alpha_{k}\right|=\beta_{i k}\left|x_{i}-x_{k}\right|$, there exists a constant $C_{3}$ which is independent of $h$ such that $\left|\beta_{i k}\right| \leq C_{3}$.

Then there exists a function $\omega_{0}(\cdot) \in B(\stackrel{\circ}{\mathscr{A}}) \cap W_{0}^{1, p}(\stackrel{\circ}{\mathscr{A}})(\stackrel{\circ}{\mathscr{A}}$ is the interior of $\mathscr{A})$, $1 \leq p \leq \infty$, such that $\omega_{0}\left(x_{i}\right)=\alpha_{i}$ for all $i \in I$, and $\left\|\omega_{0}(\cdot)\right\|_{1, p, \mathscr{A}}$ is independent of $h$.

Proof. For any $j \in J$, take a local Cartesian coordinate system with the origin at $x_{j 1}$ (see Figure 6). Let $P_{j}\left(\varsigma_{1}, \varsigma_{2}\right)=A_{j}+B_{j} \varsigma_{1}+C_{j} \zeta_{2}+D_{j \zeta_{1} \zeta_{2}}$, which satisfies

$$
\begin{aligned}
P_{j}(0,0) & =\alpha_{j 1}, & & P_{j}\left(h_{j 1}, 0\right)=\alpha_{j 2}, \\
P_{j}\left(h_{j 1}, h_{j 2}\right) & =\alpha_{j 3}, & & P_{j}\left(0, h_{j 2}\right)=\alpha_{j 4} .
\end{aligned}
$$

Then,

$$
\begin{aligned}
P_{j}\left(\varsigma_{1}, \varsigma_{2}\right)= & \alpha_{j 1}+\left\{\left(\alpha_{j 2}-\alpha_{j 1}\right) / h_{j 1}\right\} \varsigma_{1}+\left\{\left(\alpha_{j 4}-\alpha_{j 1}\right) / h_{j 2}\right\} \zeta_{2} \\
& +\left\{\left[\left(\alpha_{j 3}-\alpha_{j 2}\right)+\left(\alpha_{j 1}-\alpha_{j 4}\right)\right] / h_{j 1} h_{j 2}\right\} \zeta_{1} \varsigma_{2} .
\end{aligned}
$$

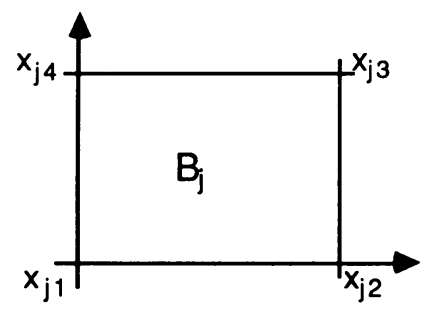

FIGURE 6

For the global coordinate system on $B_{j},\left(x_{1}, x_{2}\right)=\left(x_{j 11}+\varsigma_{1}, x_{j 12}+\varsigma_{2}\right)$, where $\left(x_{j 11}, x_{j 12}\right)$ is the coordinate for the point $x_{j 1}, 0 \leq \varsigma_{k} \leq h_{j k}, k=1,2$. On $B_{j}$, define $\mu_{j}\left(x_{1}, x_{2}\right)=\mu_{j}\left(x_{j 11}+\varsigma_{1}, x_{j 12}+\varsigma_{2}\right)=P_{j}\left(\varsigma_{1}, \varsigma_{2}\right)$. Let $\omega_{0}(\cdot)$ be a function defined on $\mathscr{A}$ in the following way:

$$
\left.\omega_{0}(x)\right|_{B_{j}}=\mu_{j}\left(x_{1}, x_{2}\right)=P_{j}\left(\varsigma_{1}, \varsigma_{2}\right) \quad \text { for all } j \in J .
$$


By finite element theory, $\omega_{0}(\cdot) \in B(\mathscr{A})$ and $\left.\omega_{0}(x)\right|_{\partial \mathscr{A}}=0, \omega_{0}$ is differentiable a.e., $\left.\partial^{\alpha} \omega_{0}(x)\right|_{B_{j}}=\partial^{\alpha} \rho_{j}$ for $|\alpha|=1$. So,

$$
\begin{aligned}
& \left|P_{j}\left(\varsigma_{1}, \varsigma_{2}\right)\right| \\
& \quad \leq\left|\alpha_{j 1}\right|+\left|\left(\alpha_{j 2}-\alpha_{j 1}\right)\right|+\left|\left(\alpha_{j 4}-\alpha_{j 1}\right)\right|+\left|\left(\alpha_{j 3}-\alpha_{j 2}\right)\right|+\left|\left(\alpha_{j 1}-\alpha_{j 4}\right)\right| \\
& \quad \leq\left|\alpha_{j 1}\right|+\left|\beta_{j_{2} j_{1}}\right| h_{j 1}+\left|\beta_{j_{4} j_{1}}\right| h_{j 2}+\left|\beta_{j_{3} j_{2}}\right| h_{j_{2}}+\left|\beta_{j_{1} j_{4}}\right| h_{j 1} \leq C_{2}+4 C_{3} \quad \forall j \in J
\end{aligned}
$$

from which

$$
\left\|\omega_{0}\right\|_{L^{\infty}(\mathscr{A})} \leq C_{2}+4 C_{3} .
$$

Meanwhile,

$$
\begin{aligned}
\partial \mu_{j} / \partial x_{1} & =\left(\partial \mu_{j} / \partial \zeta_{1}\right)\left(\partial \zeta_{1} / \partial x_{1}\right)+\left(\partial \mu_{j} / \partial_{\zeta_{2}}\right)\left(\partial_{\zeta_{2}} / \partial x_{1}\right) \\
& =\left\{\left(\alpha_{j 2}-\alpha_{j 1}\right) / h_{j 1}\right\}+\left\{\left[\left(\alpha_{j 3}-\alpha_{j 2}\right)+\left(\alpha_{j 1}-\alpha_{j 4}\right)\right] / h_{j 1} h_{j 2}\right\}_{\zeta_{2}}
\end{aligned}
$$

and so, $\left|\partial \mu_{j} / \partial x_{1}\right| \leq\left|\beta_{j_{2} j_{1}}\right|+\left|\beta_{j_{3} j_{2}}\right| C_{1}+\left|\beta_{j_{1} j_{4}}\right| C_{1} \leq C_{3}\left(1+2 C_{1}\right)$. Similarly, $\left|\partial \rho_{j} / \partial x_{2}\right| \leq C_{3}\left(1+2 C_{1}\right)$, and

$$
\left\|\partial^{\alpha} \omega_{0}\right\|_{L^{\infty}(\mathscr{A})} \leq C_{3}\left(1+2 C_{1}\right), \quad|\alpha|=1
$$

(3.11) and (3.12) imply that $\omega_{0} \in W_{0}^{1, \infty}(\mathscr{A})$. Since $\mathscr{A}$ is bounded, $\omega_{0} \in W_{0}^{1, \infty}(\mathscr{A})$ implies that $\omega_{0} \in W_{0}^{1, p}(\mathscr{A})$ for $1 \leq p<\infty$ and $\left\|\omega_{0}\right\|_{1, p, \mathscr{A}}$ depends on $C_{1}, C_{2}, C_{3}$ and $\mathscr{A}$.

LEMMA 3.12. Assume the conditions:

(1) $g(\cdot) \in W^{2,1}\left(\mathbf{R}^{2}\right) \cap W^{2, \infty}\left(\mathbf{R}^{2}\right)$ and satisfies

$$
\left|\partial^{\alpha} g(x)\right| \leq C_{1}(1+|x|)^{-4} \text { for all } x \in \mathbf{R}^{2} \text { and }|\alpha|=2
$$

(2) $h / \varepsilon \leq C_{2}$.

(3) $\left\{\alpha_{i}\right\}_{i \in I}$ satisfies (1), (2) and (3) in Lemma 3.11.

Then there exists a constant $C$ which is independent of $h$ and $\varepsilon$ such that

$$
\begin{aligned}
&\left|\sum_{i \in I} \alpha_{i}\right| S_{i}\left|\frac{\partial K * g_{\varepsilon}}{\partial x_{k}}\left(x-X^{h}\left(x_{i}, t\right)\right)\right| \leq C\left(1+\|e(t)\|_{h, \infty} / \varepsilon\right)^{2} \\
& \forall x \in \mathbf{R}^{2}, t \in[0, T], k=1,2 .
\end{aligned}
$$

Proof. [18, Chapter II, Lemma 5.6: Substitute $\omega_{0}$ in Lemma 3.11 for $\left.w_{0}\right]$.

LEMMA 3.13. Assume the conditions (1), (2) and (3) in Lemma 3.12. Then there exists a constant $C$ which is independent of $h$ and $\varepsilon$ such that for all $t \in[0, T]$ and $p \in[0, \infty],\|W(t)\|_{h, p} \leq C\|e(t)\|_{h, p}\left(1+\|e(t)\|_{h, \infty} / \varepsilon\right)^{2}$, where $W\left(X, X^{h}, t\right)$ is defined by (3.10) and $\|W(t)\|_{h, p}$ is given by Definition 3.1 . 
Proof. By (3.10) we have

$$
\begin{aligned}
\mid W & \left(X\left(x_{j}, t\right), X^{h}\left(x_{j}, t\right), t \mid\right. \\
& =\left|\sum_{i \in I}\right| S_{i}\left|\alpha_{i}\left[K * g_{\varepsilon}\left(X\left(x_{j}, t\right)-X^{h}\left(x_{i}, t\right)\right)-K * g_{\varepsilon}\left(X^{h}\left(x_{j}, t\right)-X^{h}\left(x_{i}, t\right)\right)\right]\right| \\
& =\left|\sum_{i \in I}\right| S_{i} \mid \alpha_{i}\left\{\int_{0}^{1} D K * g_{\varepsilon}\left[X\left(x_{j}, t\right)-X^{h}\left(x_{i}, t\right)+\theta\left(X^{h}\left(x_{j}, t\right)-X\left(x_{j}, t\right)\right)\right] d \theta\right\} \\
& \leq \int_{0}^{1}\left|\sum_{i \in I}\right| S_{i}\left|\alpha_{i} D K * g_{\varepsilon}\left[X\left(x_{j}, t\right)-X_{j}^{h}\left(x_{i}, t\right)+\theta\left(X^{h}\left(x_{j}, t\right)-X\left(x_{j}, t\right)\right)\right]\right| d \theta \\
& \leq C_{3}\left(1+\|e(t)\|_{h, \infty} / \varepsilon\right)^{2}\left|X^{h}\left(x_{j}, t\right)-X\left(x_{j}, t\right)\right| \quad(\operatorname{Lemma} 3.12),
\end{aligned}
$$

and hence, $\|W(t)\|_{h, p} \leq C\|e(t)\|_{h, p}\left(1+\|e(t)\|_{h, \infty} / \varepsilon\right)^{2}$.

THEOREM 3.5. Assume the conditions:

(1) $f(\cdot) \in W^{2,1}\left(\mathbf{R}^{2}\right) \cap W^{2, \infty}\left(\mathbf{R}^{2}\right)$ and satisfies

$$
\left|\partial^{\alpha} f(x)\right| \leq C_{1}(1+|x|)^{-4} \quad \text { for all } x \in \mathbf{R}^{2} \text { and }|\alpha|=2 .
$$

(2) $h / \varepsilon \leq C_{2}$.

Then there exists a constant $C=C\left(w_{0}, p, T\right)$ such that

$\left\|W_{11}(t)\right\|_{h, p} \leq C\left(1+\|e(t)\|_{h, \infty} / \varepsilon\right)^{2}\|e(t)\|_{h, p} \quad$ for all $t \in[0, T], p \in[1, \infty]$.

Proof. By definition,

$$
W_{11}\left(X, X^{h}, t\right)=\sum_{i \in I} c_{i}\left[K * f_{\varepsilon}\left(X-X^{h}\left(x_{i}, t\right)\right)-K * f_{\varepsilon}\left(X^{h}-X^{h}\left(x_{i}, t\right)\right)\right],
$$

where $c_{i}=\left|S_{i}\right|\left(w_{0}\left(x_{i}\right)+H_{i 1} w_{0 x_{1}}\left(x_{i}\right)+H_{i 2} w_{0 x_{2}}\left(x_{i}\right)\right)$. Let $\alpha_{i}=w_{0}\left(x_{i}\right)+H_{i 1} w_{0 x_{1}}\left(x_{i}\right)$ $+H_{i 2} w_{0 x_{2}}\left(x_{i}\right)$; then $\left|\alpha_{i}\right| \leq C_{3}\left\|w_{0}\right\|_{W^{1, \infty}\left(\mathbf{R}^{2}\right)}$ for all $i \in I$. For two adjacent $x_{i}$ and $x_{k}$ with $i, k \in I$ we have

$$
\begin{aligned}
\left|\alpha_{i}-\alpha_{k}\right|= & \mid w_{0}\left(x_{i}\right)-w_{0}\left(x_{k}\right)+H_{i 1}\left(w_{0 x_{1}}\left(x_{i}\right)-w_{0 x_{1}}\left(x_{k}\right)\right) \\
& +H_{i 2}\left(w_{0 x_{2}}\left(x_{i}\right)-w_{0 x_{2}}\left(x_{k}\right)\right)+w_{0 x_{1}}\left(x_{k}\right)\left(H_{i 1}-H_{k 1}\right) \\
& +w_{0 x_{2}}\left(x_{k}\right)\left(H_{i 2}-H_{k 2}\right) \mid \\
= & \mid D w_{0}\left(x_{i k}\right) \cdot\left(x_{i}-x_{k}\right)+H_{i 1} D w_{0 x_{1}}\left(y_{i k}\right) \cdot\left(x_{i}-x_{k}\right) \\
& +H_{i 2} D w_{0 x_{2}}\left(z_{i k}\right) \cdot\left(x_{i}-x_{k}\right)+w_{0 x_{1}}\left(x_{k}\right)\left(H_{i 1}-H_{k 1}\right) \\
\leq & \quad+w_{0 x_{2}}\left(x_{k}\right)\left(H_{i 2}-H_{k 2}\right) \mid \\
\leq & C_{4}\left\|w_{0}\right\|_{W^{2, \infty}\left(\mathbf{R}^{2}\right)}\left|x_{i}-x_{k}\right|, \quad
\end{aligned}
$$

where $x_{i k}, y_{i k}$ and $z_{i k}$ are intermediate points between $x_{i}$ and $x_{k}$. Thus, by Lemma 3.13 ,

$\left\|W_{11}(t)\right\|_{h, p} \leq C_{5}\|e(t)\|_{h, p}\left(1+\|e(t)\|_{h, \infty} / \varepsilon\right)^{2} \quad \forall t \in[0, T], p \in[0, \infty]$.

For the estimation of $W_{21}\left(X, X^{h}, t\right)$ and $W_{22}\left(X, X^{h}, t\right)$, we have the following result. 
THEOREM 3.6. Assume the following conditions:

(1) $f(\cdot) \in W^{2,1}\left(\mathbf{R}^{2}\right) \cap W^{2, \infty}\left(\mathbf{R}^{2}\right)$ and satisfies

$$
|x|^{4}\left|\partial^{\alpha} f(x)\right| \leq C_{1} \quad \text { for all } x \in \mathbf{R}^{2} \text { and }|\alpha|=2 \text {. }
$$

(2) $h \leq C_{2} \varepsilon^{2}$.

Let $T_{\varepsilon}^{*}=\left\{t \in[0, T] \mid\|e(t)\|_{h, \infty} \leq M_{1} \varepsilon,\|E(t)\|_{h, \infty} \leq M_{2}\right\}$, where $M_{1}$ and $M_{2}$ are two arbitrary constants. Then there exists a constant $C=C\left(w_{0}, T, p\right)$ such that

$\left\|W_{21}(t)\right\|_{h, p}+\left\|W_{22}(t)\right\|_{h, p} \leq C\|e(t)\|_{h, p} \quad$ for all $t \in\left[0, T_{\varepsilon}^{*}\right]$ and $p \in(1, \infty)$.

Proof. By definition,

$$
\begin{aligned}
W_{21}\left(X, X^{h}, t\right)=-\sum_{i \in I} d_{1 i}(t)\left[K_{\varepsilon x_{1}}\left(X-X^{h}\left(x_{i}, t\right)\right)-K_{\varepsilon x_{1}}\left(X^{h}-X^{h}\left(x_{i}, t\right)\right)\right] \\
=-\sum_{i \in I} \frac{d_{1 i}(t)}{\varepsilon}\left[K * f_{x_{1} \varepsilon}\left(X-X^{h}\left(x_{i}, t\right)\right)-K * f_{x_{1} \varepsilon}\left(X^{h}-X^{h}\left(x_{i}, t\right)\right)\right] \\
=\sum_{i \in I} \frac{d_{1 i}(t)}{\varepsilon}\left[\int_{0}^{1} D K * f_{x_{1} \varepsilon}\left(X-X^{h}\left(x_{i}, t\right)+\theta\left(X-X^{h}\right)\right) d \theta\right] \cdot\left(X^{h}-X\right),
\end{aligned}
$$

and

$$
\begin{array}{r}
W_{21}\left(X\left(x_{j}, t\right), X^{h}\left(x_{j}, t\right), t\right) \\
=\sum_{i \in I} \frac{d_{1 i}(t)}{\varepsilon}\left[\int_{0}^{1} D K * f_{x_{1} \varepsilon}\left(X\left(x_{j}, t\right)-X^{h}\left(x_{i}, t\right)+\theta\left(X\left(x_{j}, t\right)-X^{h}\left(x_{j}, t\right)\right)\right) d \theta\right] \\
\cdot\left(X^{h}\left(x_{j}, t\right)-X\left(x_{j}, t\right)\right) .
\end{array}
$$

So,

$$
\begin{aligned}
& \left|W_{21}\left(X\left(x_{j}, t\right), X^{h}\left(x_{j}, t\right), t\right)\right| \\
& \leq\left|X^{h}\left(x_{j}, t\right)-X\left(x_{j}, t\right)\right| \\
& \quad \times \sum_{i \in I} \frac{\left|d_{1 i}(t)\right|}{\varepsilon} \max _{0 \leq \theta \leq 1}\left|D K * f_{x_{1} \varepsilon}\left(X\left(x_{j}, t\right)-X^{h}\left(x_{i}, t\right)+\theta\left(X\left(x_{j}, t\right)-X^{h}\left(x_{i}, t\right)\right)\right)\right| \\
& =\left|X^{h}\left(x_{j}, t\right)-X\left(x_{j}, t\right)\right| \\
& \quad \times \sum_{i \in I} \frac{\left|d_{1 i}(t)\right|}{\varepsilon} \max _{0 \leq \theta \leq 1} \mid D K * f_{x_{1} \varepsilon}\left(X\left(x_{j}, t\right)+\theta\left(X\left(x_{j}, t\right)-X^{h}\left(x_{j}, t\right)\right)\right. \\
&
\end{aligned}
$$

where $y_{i}=X\left(x_{i}, t\right)-X^{h}\left(x_{i}, t\right)$ and $\left|y_{i}\right| \leq\|e(t)\|_{h, \infty}$. By the definition of $d_{1 i}(t)$,

$$
d_{1 i}(t) / \varepsilon=-\left[M_{11}^{h}\left(x_{i}, t\right) H_{i 1}+M_{12}^{h}\left(x_{i}, t\right) H_{i 2}\right] w_{0}\left(x_{i}\right)\left|S_{i}\right| / \varepsilon .
$$

Since for $t \in\left[0, T_{\varepsilon}^{*}\right],\|E(t)\|_{h, \infty} \leq M_{2}$, and $\|M(\cdot, t)\|_{L^{\infty}\left(\mathbf{R}^{2}\right)}$ is bounded for $t \in$ $\left[0, T_{\varepsilon}^{*}\right]$, we have $\left|M^{h}\left(x_{i}, t\right)\right| \leq C_{6}$ for all $i \in I$ and $t \in\left[0, T_{\varepsilon}^{*}\right]$. Therefore,

$$
\left|M_{11}^{h}\left(x_{i}, t\right) H_{i 1}+M_{12}^{h}\left(x_{i}, t\right) H_{i 2}\right| / \varepsilon \leq C_{7} \varepsilon \quad \forall i \in I \text { and } t \in\left[0, T_{\varepsilon}^{*}\right],
$$

because $h \leq C_{2} \varepsilon^{2}, H_{i 1}=O(h)$ and $H_{i 2}=O(h)$. Hence,

$$
\left|d_{1 i}(t) / \varepsilon\right| \leq C_{7} \varepsilon\left\|w_{0}\right\|_{L^{\infty}\left(\mathbf{R}^{2}\right)}\left|S_{i}\right| \quad \text { for all } i \in I .
$$


Using Lemma 3.9 and Lemma 3.10 with $|\beta|=1$,

$$
\begin{aligned}
& \left|W_{21}\left(X\left(x_{j}, t\right), X^{h}\left(x_{j}, t\right), t\right)\right| \\
& \leq|| X^{h}\left(x_{j}, t\right)-X\left(x_{j}, t\right) \mid \\
& \quad \times \sum_{i \in I} \frac{\left|d_{1 i}(t)\right|}{\varepsilon} \max _{0 \leq \theta \leq 1} \mid D K * f_{x_{1} \varepsilon}\left(X\left(x_{j}, t\right)+\theta\left(X\left(x_{j}, t\right)-X^{h}\left(x_{j}, t\right)\right)\right. \\
& \quad \leq\left|X^{h}\left(x_{j}, t\right)-X\left(x_{j}, t\right)\right| C_{7} \varepsilon\left\|w_{0}\right\|_{L^{\infty}\left(\mathbf{R}^{2}\right)} C_{8}|\log \varepsilon| \leq C_{9}\left|X^{h}\left(x_{j}, t\right)-X\left(x_{j}, t\right)\right| .
\end{aligned}
$$

This implies that $\left\|W_{21}(t)\right\|_{h, p} \leq C_{9}\|e(t)\|_{h, p}$ for all $t \in\left[0, T_{\varepsilon}^{*}\right], p \in(1, \infty)$. Similarly, $\left\|W_{22}(t)\right\|_{h, p} \leq C_{10}\|e(t)\|_{h, p}$ for all $t \in\left[0, T_{\varepsilon}^{*}\right], p \in(1, \infty)$.

The Error Bounds. Now we will give the error estimate for the 2-D vortex method constructed previously.

THEOREM 3.7 [Summary]. Assume the following conditions:

(1) (i), (ii) and (iii) of Theorem 3.1.

(2) $f(\cdot) \in W^{4,1}\left(\mathbf{R}^{2}\right) \cap W^{4, \infty}\left(\mathbf{R}^{2}\right)$ and there exist constants $C_{1}, C_{2}$ and $\gamma>2$ such that

$$
\begin{array}{ll}
\left|\partial^{\alpha} f(x)\right| \leq C_{1}(1+|x|)^{-\gamma}, & x \in \mathbf{R}^{2},|\alpha|=0,1 \\
\left|\partial^{\alpha} f(x)\right| \leq C_{2}(1+|x|)^{-4}, & x \in \mathbf{R}^{2},|\alpha|=2 .
\end{array}
$$

(3) There exists a constant $C_{3}>0$ such that $h \leq C_{3} \varepsilon^{2}$.

Then, for $2<p<\infty$, there exists a constant $C=C\left(p, T, s, w_{0}\right)$ such that

$$
\left\|\frac{d}{d t} e(t)\right\|_{h, p} \leq C\left(\varepsilon^{k}+\frac{h^{4}}{\varepsilon^{3+s}}+\|e(t)\|_{h, p}+\varepsilon\|E(t)\|_{h, p}\right) \quad \forall t \in\left[0, T_{\varepsilon}^{*}\right],
$$

where $s>0$ is an arbitrary number.

Proof. This result is the content of Theorems 3.1-3.6.

Since the error bound for $d\left\{e\left(X, X^{h}, t\right)\right\} / d t$ involves the term $\varepsilon\|E(t)\|_{h, p}$, we now need to analyze $d\left\{\varepsilon E\left(X, X^{h}, t\right)\right\} / d t$ :

$$
\frac{d}{d t} \varepsilon E\left(X, X^{h}, t\right)=\frac{d}{d t} \varepsilon\left\{M(x, t)-M^{h}(x, t)\right\} .
$$

According to the systems of ordinary differential equations for $M$ and $M^{h}$,

$$
\begin{aligned}
\varepsilon \frac{d}{d t}\{ & \left.M(x, t)-M^{h}(x, t)\right\}=\varepsilon\left\{\nabla u(X, t) M(x, t)-\nabla \mathscr{U}_{\varepsilon}^{h}\left(X^{h}, t\right) M^{h}(x, t)\right\} \\
= & \varepsilon\left\{\left(\nabla u(X, t)-\nabla \mathscr{U}_{\varepsilon}^{h}\left(X^{h}, t\right)\right) M(x, t)+\nabla \mathscr{U}_{\varepsilon}^{h}\left(X^{h}, t\right)\left(M(x, t)-M^{h}(x, t)\right)\right\} \\
= & \varepsilon\left\{\left(\nabla u(X, t)-\nabla \mathscr{U}_{\varepsilon}^{h}\left(X^{h}, t\right)\right) M(x, t)\right. \\
& \quad+\left(\nabla \mathscr{U}_{\varepsilon}^{h}\left(X^{h}, t\right)-\nabla u(X, t)\right)\left(M(x, t)-M^{h}(x, t)\right) \\
& \left.+\nabla u(X, t)\left(M(x, t)-M^{h}(x, t)\right)\right\} .
\end{aligned}
$$


Since we assume that the true solution is smooth,

$$
\begin{gathered}
\left\{\sum_{i \in I}\left|\nabla u\left(X\left(x_{i}, t\right), t\right)\left(M\left(x_{i}, t\right)-M^{h}\left(x_{i}, t\right)\right)\right|^{p} h^{2}\right\}^{1 / p} \leq C_{1}\|E(t)\|_{h, p} \\
\left\{\sum_{i \in I}\left|\left(\nabla \mathscr{U}_{\varepsilon}^{h}\left(X^{h}\left(x_{i}, t\right), t\right)-\nabla u\left(X\left(x_{i}, t\right), t\right)\right)\left(M\left(x_{i}, t\right)-M^{h}\left(x_{i}, t\right)\right)\right|^{p} h^{2}\right\}^{1 / p} \\
\leq\|E(t)\|_{h, \infty}\left\{\sum_{i \in I}\left|\nabla \mathscr{U}_{\varepsilon}^{h}\left(X^{h}\left(x_{i}, t\right), t\right)-\nabla u\left(X\left(x_{i}, t\right), t\right)\right|^{p} h^{2}\right\}^{1 / p}
\end{gathered}
$$

and

$$
\begin{aligned}
& \left\{\sum_{i \in I}\left|\left(\nabla \mathscr{U}_{\varepsilon}^{h}\left(X^{h}\left(x_{i}, t\right), t\right)-\nabla u\left(X\left(x_{i}, t\right), t\right)\right) M\left(x_{i}, t\right)\right|^{p} h^{2}\right\}^{1 / p} \\
& \quad \leq\|M(\cdot, t)\|_{L^{\infty}\left(\mathbf{R}^{2}\right)}\left\{\sum_{i \in I}\left|\nabla \mathscr{U}_{\varepsilon}^{h}\left(X^{h}\left(x_{i}, t\right), t\right)-\nabla u\left(X\left(x_{i}, t\right), t\right)\right|^{p} h^{2}\right\}^{1 / p} .
\end{aligned}
$$

So, for $t \in\left[0, T_{\varepsilon}^{*}\right]$, Eqs. (3.13), (3.14) and (3.15) imply that

$$
\begin{aligned}
& \left\|\frac{d}{d t} \varepsilon E(t)\right\|_{h, p} \\
& \quad \leq C_{2}\left(\varepsilon\|E(t)\|_{h, p}+\varepsilon\left\{\sum_{i \in I}\left|\nabla u\left(X\left(x_{i}, t\right), t\right)-\nabla \mathscr{U}_{\varepsilon}^{h}\left(X^{h}\left(x_{i}, t\right), t\right)\right|^{p} h^{2}\right\}^{1 / p}\right) .
\end{aligned}
$$

By norm equivalence,

$$
\begin{gathered}
\left\{\sum_{i \in I}\left|\nabla u\left(X\left(x_{i}, t\right), t\right)-\nabla \mathscr{U}_{\varepsilon}^{h}\left(X^{h}\left(x_{i}, t\right), t\right)\right|^{p} h^{2}\right\}^{1 / p} \\
\leq C_{3}\left\{\left[\sum_{i \in I}\left|\frac{\partial u}{\partial x_{1}}\left(X\left(x_{i}, t\right), t\right)-\frac{\partial \mathscr{U}_{\varepsilon}^{h}}{\partial x_{1}}\left(X^{h}\left(x_{i}, t\right), t\right)\right|^{p} h^{2}\right]^{1 / p}\right. \\
\left.+\left[\sum_{i \in I}\left|\frac{\partial u}{\partial x_{2}}\left(X\left(x_{i}, t\right), t\right)-\frac{\partial \mathscr{U}_{\varepsilon}^{h}}{\partial x_{2}}\left(X^{h}\left(x_{i}, t\right), t\right)\right|^{p} h^{2}\right]^{1 / p}\right\}, \\
\varepsilon\left(\frac{\partial u}{\partial x_{k}}(X, t)-\frac{\partial \mathscr{U}_{\varepsilon}^{h}}{\partial x_{k}}\left(X^{h}, t\right)\right)=\varepsilon\left(K * w_{x_{k}}(X, t)-\frac{\partial \mathscr{U}_{\varepsilon}^{h}}{\partial x_{k}}\left(X^{h}, t\right)\right) \quad(k=1 \text { or } 2) \\
=\varepsilon\left\{\left[K * w_{x_{k}}(X, t)-K_{\varepsilon} * w_{x_{k}}(X, t)\right]+\left[K_{\varepsilon} * w_{x_{k}}(X, t)-\frac{\partial \mathscr{U}_{\varepsilon}^{h}}{\partial x_{k}}\left(X^{h}, t\right)\right]\right\} \\
=\varepsilon\left\{\mathrm{I}_{1}+\mathrm{I}_{2}\right\} .
\end{gathered}
$$

$I_{1}$ can be bounded by using the method of Theorem 3.1 , substituting $w_{x_{1}}(\cdot, t)$ or $w_{x_{2}}(\cdot, t)$ for $w(\cdot, t)$ in Theorem 3.1. Considering the second term $\mathrm{I}_{2}$, for $k=1,2$ we 
have

$$
\begin{aligned}
& \mathrm{I}_{2}= \varepsilon\left(K_{\varepsilon} * w_{x_{k}}(X, t)-\frac{\partial \mathscr{U}_{\varepsilon}^{h}}{\partial x_{k}}\left(X^{h}, t\right)\right) \\
&=\varepsilon\left\{\left(\partial K_{\varepsilon} / \partial x_{k}\right) * w(X, t)\right. \\
& \quad-\sum_{i \in I}\left[c_{i} \frac{\partial K_{\varepsilon}}{\partial x_{k}}\left(X^{h}-X^{h}\left(x_{i}, t\right)\right)-d_{1 i}(t) \frac{\partial^{2} K_{\varepsilon}}{\partial x_{k} \partial x_{1}}\left(X^{h}-X^{h}\left(x_{i}, t\right)\right)\right. \\
&=(\left.\left.-d_{2 i}(t) \frac{\partial^{2} K_{\varepsilon}}{\partial x_{k} \partial x_{2}}\left(X^{h}-X^{h}\left(x_{i}, t\right)\right)\right]\right\} \\
&-\sum_{i \in I}\left[c_{x_{k} \varepsilon} K * w_{x_{k} \varepsilon}\left(X^{h}-X^{h}\left(x_{i}, t\right)\right)\right. \\
&-d_{1 i}(t)\left(K * f_{x_{k} \varepsilon}\right)_{x_{1}}\left(X^{h}-X^{h}\left(x_{i}, t\right)\right) \\
&\left.-d_{2 i}(t)\left(K * f_{x_{k} \varepsilon}\right)_{x_{2}}\left(X^{h}-X^{h}\left(x_{i}, t\right)\right)\right],
\end{aligned}
$$

where $f_{x_{k} \varepsilon}(x)=f_{x_{k}}(x / \varepsilon) / \varepsilon^{2}$. By substituting $f_{x_{k} \varepsilon}(\cdot)$ for $f_{\varepsilon}(\cdot)$ in Theorem 3.7, we obtain the following result.

THEOREM 3.8 [Corollary of Theorem 3.7]. Assume the conditions:

(1) (i), (ii) and (iii) of Theorem 3.1 .

(2) $f(\cdot) \in W^{5,1}\left(\mathbf{R}^{2}\right) \cap W^{5, \infty}\left(\mathbf{R}^{2}\right)$. There exist constants $C_{1}, C_{2}$ and $\gamma>2$ such that

$$
\begin{array}{ll}
\left|\partial^{\alpha} f(x)\right| \leq C_{1}(1+|x|)^{-\gamma}, & x \in \mathbf{R}^{2},|\alpha|=0,1 \\
\left|\partial^{\alpha} f(x)\right| \leq C_{2}(1+|x|)^{-4}, & x \in \mathbf{R}^{2},|\alpha|=2,3 .
\end{array}
$$

(3) There exists a constant $C_{3}>0$ such that $h \leq C_{3} \varepsilon^{2}$.

Then, for $2<p<\infty$, there exists a constant $C=C\left(p, T, s, w_{0}\right)$ such that

$$
\begin{aligned}
& \left\|\frac{d}{d t} e(t)\right\|_{h, p}+\varepsilon\left\|\frac{d}{d t} E(t)\right\|_{h, p} \\
& \leq C\left(\varepsilon^{k}+\frac{h^{4}}{\varepsilon^{3+s}}+\|e(t)\|_{h, p}+\varepsilon\|E(t)\|_{h, p}\right) \quad \forall t \in\left[0, T_{\varepsilon}^{*}\right],
\end{aligned}
$$

where $s>0$ is an arbitrary number.

For the main estimate, the Gronwall inequality is needed.

LEMMA 3.15 [Gronwall inequality]. Let $G: \mathbf{R} \rightarrow \mathbf{R}$ be a smooth function. Let $\|\cdot\|$ be a norm on $\mathbf{R}^{n}$ and let e be a continuously differentiable $n$-vector function on $\left[0, T^{*}\right]$ such that $e(0)=0$ and $\|d e(t) / d t\| \leq G(\|e(t)\|)$. Let $y$ be the real-valued function defined by $d y(t) / d t=G(y(t))$ and $y(0)=0$. Then for $t \in\left[0, T^{*}\right],\|e(t)\| \leq$ $y(t)$.

Proof. See [11, Section I.6].

THEOREM 3.9 [The main estimate]. Assume the following conditions:

(1) Conditions (1) and (2) of Theorem 3.8 with $k \geq 2$. 
(2) There exist three constants $C_{3}>0, \alpha$ and $\beta$ such that

$$
\alpha \geq \beta \geq 2 \quad \text { and } \quad C_{3}^{-1} \varepsilon^{\alpha} \leq h \leq C_{3} \varepsilon^{\beta} .
$$

Then we have the following results:

(a) For $2<p<\infty$, there exists a constant $C_{s}=C_{s}\left(p, w_{0}, T\right)$ such that

$$
\begin{gathered}
\|e(t)\|_{h, p}+\varepsilon\|E(t)\|_{h, p} \leq C_{s}\left(\varepsilon^{k}+\frac{h^{4}}{\varepsilon^{3+s}}\right), \\
\left\|u(t)-\mathscr{U}_{\varepsilon}^{h}(t)\right\|_{h, p}+\varepsilon\left\|\nabla u(t)-\nabla \mathscr{U}_{\varepsilon}^{h}(t)\right\|_{h, p} \leq C_{s}\left(\varepsilon^{k}+\frac{h^{4}}{\varepsilon^{3+s}}\right) .
\end{gathered}
$$

(b) There exists a constant $C_{s}=C_{s}\left(p, w_{0}, T\right)$ such that

$$
\begin{gathered}
\|e(t)\|_{h, \infty}+\varepsilon\|E(t)\|_{h, \infty} \leq \frac{C_{s}}{\varepsilon^{s}}\left(\varepsilon^{k}+\frac{h^{4}}{\varepsilon^{3}}\right), \\
\left\|u(t)-\mathscr{U}_{\varepsilon}^{h}(t)\right\|_{h, \infty}+\varepsilon\left\|\nabla u(t)-\nabla \mathscr{U}_{\varepsilon}^{h}(t)\right\|_{h, \infty} \\
\leq \frac{C_{s}}{\varepsilon^{s}}\left(\varepsilon^{k}+\frac{h^{4}}{\varepsilon^{3}}\right) \quad \forall t \in[0, T],
\end{gathered}
$$

where $s>0$ is an arbitrary number.

Proof. By Theorem 3.8, for $p \in(0, \infty)$ there exists a constant $C_{1 s}=C_{1 s}\left(p, w_{0}, T\right)$ such that for all $t \in\left[0, T_{\varepsilon}^{*}\right]$,

$$
\left\|\frac{d}{d t} e(t)\right\|_{h, p}+\varepsilon\left\|\frac{d}{d t} E(t)\right\|_{h, p} \leq C_{1 s}\left(\varepsilon^{k}+\frac{h^{4}}{\varepsilon^{3+s}}+\|e(t)\|_{h, p}+\varepsilon\|E(t)\|_{h, p}\right)
$$

where $s>0$ is an arbitrary number. For $(3.16)$, let $e\left(X, X^{h}, t\right)=\left(e\left(X, X^{h}, t\right)\right.$, $\left.E\left(X, X^{h}, t\right)\right)$ and define $\|e(t)\|=\|e(t)\|_{h, p}+\varepsilon\|E(t)\|_{h, p}$. Then,

$$
\|d e(t) / d t\| \leq C_{1 s}\left(\varepsilon^{k}+\frac{h^{4}}{\varepsilon^{3+s}}+\|e(t)\|\right) \quad \forall t \in\left[0, T_{\varepsilon}^{*}\right] .
$$

Define $G: \mathbf{R} \rightarrow \mathbf{R}$ by setting

$$
G(a)=C_{1 s}\left(\varepsilon^{k}+\frac{h^{4}}{\varepsilon^{3+s}}+a\right)=C_{1 s}\left(a+\varepsilon_{1}\right) .
$$

Then, $\|d e(t) / d t\| \leq G(\|e(t)\|)$ by (3.20). Solve the initial value problem

$$
d y(t) / d t=G(y(t))=C_{1 s}\left(y(t)+\varepsilon_{1}\right), \quad y(0)=0
$$

to get

$$
y(t)=\left(\exp \left(C_{1 s} t\right)-1\right) \varepsilon_{1}
$$

By Lemma 3.15,

$$
\|e(t)\| \leq y(t) \leq\left(\exp \left(C_{1 s} T\right)-1\right)\left(\varepsilon^{k}+\frac{h^{4}}{\varepsilon^{3+s}}\right) \quad \text { for all } t \in\left[0, T_{\varepsilon}^{*}\right] .
$$

So,

$$
\|e(t)\|_{h, p}+\varepsilon\|E(t)\|_{h, p} \leq C_{s}\left(\varepsilon^{k}+\frac{h^{4}}{\varepsilon^{3+s}}\right) \quad \forall t \in\left[0, T_{\varepsilon}^{*}\right], 2<p<\infty .
$$

The above result implies (3.16) for $t \in\left[0, T_{\varepsilon}^{*}\right]$, and (3.17) follows from (3.16) and (3.20). For (3.18) and (3.19), consider any $p \in(2, \infty)$ and note that

$$
\|e(t)\|_{h, p}+\varepsilon\|E(t)\|_{h, p} \geq h^{2 / p}\left(\|e(t)\|_{h, \infty}+\varepsilon\|E(t)\|_{h, \infty}\right) .
$$


Then,

$$
\begin{aligned}
& \|e(t)\|_{h, \infty}+\varepsilon\|E(t)\|_{h, \infty} \leq h^{-2 / p}\left(\|e(t)\|_{h, p}+\varepsilon\|E(t)\|_{h, p}\right) \\
& \quad \leq \frac{1}{C_{3}^{-1} \varepsilon^{2 \alpha / p}} C_{s}\left(\varepsilon^{k}+\frac{h^{4}}{\varepsilon^{3+s}}\right) \\
& \quad \leq \frac{1}{C_{3}^{-1} \varepsilon^{2 \alpha / p+s}} C_{s}\left(\varepsilon^{k}+\frac{h^{4}}{\varepsilon^{3}}\right)=\frac{C s^{\prime}}{\varepsilon^{s^{\prime}}}\left(\varepsilon^{k}+\frac{h^{4}}{\varepsilon^{3}}\right) \quad \forall t \in\left[0, T_{\varepsilon}^{*}\right] .
\end{aligned}
$$

By the same procedure we can show that

$$
\left\|u(t)-\mathscr{U}_{\varepsilon}^{h}(t)\right\|_{h, \infty}+\varepsilon\left\|\nabla u(t)-\nabla \mathscr{U}_{\varepsilon}^{h}(t)\right\|_{h, \infty} \leq \frac{C s^{\prime}}{\varepsilon^{s^{\prime}}}\left(\varepsilon^{k}+\frac{h^{4}}{\varepsilon^{3}}\right) \quad \forall t \in\left[0, T_{\varepsilon}^{*}\right],
$$

so that (3.18) and (3.19) are satisfied for all $t \in\left[0, T_{\varepsilon}^{*}\right]$.

The remaining problem is to prove that $T_{\varepsilon}^{*}=T$. Recall that

$$
T_{\varepsilon}^{*}=\max \left\{t \in[0, T] \mid\|e(t)\|_{h, \infty} \leq M_{1} \varepsilon \text { and }\|e(t)\|_{h, \infty} \leq M_{2}\right\} .
$$

Since $u, \mathscr{U}_{\varepsilon}^{h}, \nabla u$ and $\nabla \mathscr{U}_{\varepsilon}^{h}$ are continuous and bounded uniformly for $(x, t) \in \Omega \times$ $[0, T]$ and $e\left(X, X^{h}, 0\right)=0$, if $T_{\varepsilon}^{*}<T$, then $\left\|e\left(T_{\varepsilon}^{*}\right)\right\|_{h, \infty}=M_{1} \varepsilon$ or $\left\|E\left(T_{\varepsilon}^{*}\right)\right\|_{h, \infty}=$ $M_{2}$. But, by $(3.21)$,

$$
\|e(t)\|_{h, \infty}+\varepsilon\|E(t)\|_{h, \infty} \leq \frac{1}{C_{3}^{-1} \varepsilon^{2 \alpha / p}} C_{s}\left(\varepsilon^{k}+\frac{h^{4}}{\varepsilon^{3+s}}\right) \quad \text { with } k \geq 2 .
$$

Choose $p$ so large that $k-(2 \alpha / p)>1$. Then,

$$
\|e(t)\|_{h, \infty}+\varepsilon\|E(t)\|_{h, \infty} \leq \max \left(M_{1}, M_{2}\right) \varepsilon^{k-(2 \alpha / p)}
$$

for suitable $\varepsilon$ and $h$, for all $t \in\left[0, T_{\varepsilon}^{*}\right]$. This is a contradiction, and it follows that $T_{\varepsilon}^{*}=T$.

Remark. The result of Theorem 3.9 may be compared with the analogous result, using simple $\delta$ functions. For the latter case, the error bound would be $C_{s}\left(\varepsilon^{k}+h^{2} / \varepsilon^{s+1}\right)$. Hence, substantial improvements can be obtained by suitable choices for $\varepsilon$. Some numerical results for this algorithm are given in [8].

4. Conclusion. A higher-order vortex algorithm is defined for two-dimensional incompressible inviscid flows. This algorithm uses gradients of $\delta$ functions (vortex dipoles) in addition to the usual $\delta$ functions (point vortices) with appropriate smoothing. Error estimates are proved, demonstrating the higher orders of convergence on arbitrary (graded) meshes for assignment of the initial vorticity distribution.

\footnotetext{
Department of Mathematics

Carnegie Mellon University

Pittsburgh, Pennsylvania 15213

E-mail: 1044rn0m@vb.cc.cmu.edu
}

1. C. Anderson \& C. Greengard, "On vortex methods," SIAM J. Numer. Anal., v. 22, 1985, pp. 413-440.

2. J. T. BeAlE \& A. MAJdA, "Vortex methods I: Convergence in three dimensions," Math. Comp., v. 39, 1982, pp. 1-27.

3. J. T. BEALE \& A. MAJDA, "Vortex methods II: Higher order accuracy in two and three dimensions," Math. Comp., v. 39, 1982, pp. 29-52. 
4. J. T. BEALE \& A. MAJDA, "Rates of convergence for viscous splitting of the Navier-Stokes equations," Math. Comp., v. 37, 1981, pp. 243-259.

5. G. H. Cottet, Méthodes Particulaires pour l'équation d'Euler dans le Plan, Thèse de 3ème cycle, Université Paris VI, 1982.

6. A. J. ChORIN, "Numerical study of slightly viscous flow," J. Fluid Mech., v. 57, 1973, pp. 785-796.

7. A. J. Chorin \& J. MARsden, A Mathematical Introduction to Fluid Mechanics, SpringerVerlag, New York, 1979.

8. S. CHOUDHURY \& R. A. NiCOLAIDES, "Vortex multipole methods for viscous incompressible flows," 10th International Conf. on Numerical Methods in Fluid Dynamics (F. G. Zhuang and Y. L. Zhu, eds.), Lecture Notes in Phys., vol. 264, Springer-Verlag, Berlin, 1986.

9. P. G. Ciarlet, The Finite Element Method for Elliptic Problems, North-Holland, Amsterdam, 1978.

10. O. H. HALD, "Convergence of vortex methods for Euler's equations. II," SIAM J. Numer. Anal., v. 16, 1979, pp. 726-755.

11. J. K. HALE, Ordinary Differential Equations, Wiley Interscience, New York, 1969.

12. F. H. HARLOW, "The particle in cell computing method for fluid dynamics," Methods in Computational Physics (B. Alder, S. Fernbach \& M. Rotenberg, eds.), Vol. 3, Academic Press, New York, 1964.

13. R. W. Hockney \& J. W. EAstwood, Computer Simulation Using Particles, McGraw-Hill, New York, 1981.

14. T. KAтO, "Nonstationary flows of viscous and ideal fluids in $R^{3}$," J. Funct. Anal., v. 9, 1972, pp. 296-305.

15. A. LeOnARD, "Vortex methods for flow simulations," J. Comput. Phys., v. 37, 1980, pp. 289-335.

16. F. J. MCGRAth, "Nonstationary plane flow of viscous and ideal fluids," Arch. Rational Mech. Anal., v. 27, 1968, pp. 328-348.

17. R. A. NiCOLAIDES, "Construction of higher order accurate vortex and particle methods," Appl. Numer. Math., v. 2, 1986, pp. 313-320.

18. P. A. RAviart, "An analysis of particle methods," Numerical Methods in Fluid Dynamics, Como, July, 1983.

19. R.TEMAM, "Local existence of $C^{\infty}$ solutions of the Euler equations of incompressible perfect fluids," Turbulence and Navier-Stokes Equations (R. Temam, ed.), Lecture Notes in Math., vol. 565, Springer-Verlag, Berlin, 1976. 